\title{
A systematic review of waist-to-height ratio as a screening tool for the prediction of cardiovascular disease and diabetes: $0 \cdot 5$ could be a suitable global boundary value
}

\author{
Lucy M. Browning ${ }^{1}$, Shiun Dong $\mathrm{Hsieh}^{2}$ and Margaret Ashwell ${ }^{1,3 *}$ \\ ${ }^{1}$ Ashwell Associates Ltd, Ashwell Street, Ashwell, Hertfordshire SG7 5PZ, UK \\ ${ }^{2}$ Health Management Centre, Toranomon Hospital, Toranomon, Minato-Ku, Tokyo, Japan \\ ${ }^{3}$ Oxford Brookes University, Oxford OX3 OBP, UK
}

\begin{abstract}
This systematic review collated seventy-eight studies exploring waist-to-height ratio (WHtR) and waist circumference (WC) or BMI as predictors of diabetes and CVD, published in English between 1950 and 2008. Twenty-two prospective analyses showed that WHtR and WC were significant predictors of these cardiometabolic outcomes more often than BMI, with similar OR, sometimes being significant predictors after adjustment for BMI. Observations from crosssectional analyses, forty-four in adults, thirteen in children, supported these predictions. Receiver operator characteristic (ROC) analysis revealed mean area under ROC (AUROC) values of 0.704, 0.693 and 0.671 for WHtR, WC and BMI, respectively. Mean boundary values for WHtR, covering all cardiometabolic outcomes, from studies in fourteen different countries and including Caucasian, Asian and Central American subjects, were 0.50 for men and 0.50 for women. WHtR and WC are therefore similar predictors of diabetes and CVD, both being stronger than, and independent of, BMI. To make firmer statistical comparison, a meta-analysis is required. The AUROC analyses indicate that WHtR may be a more useful global clinical screening tool than WC, with a weighted mean boundary value of $0 \cdot 5$, supporting the simple public health message 'keep your waist circumference to less than half your height'.
\end{abstract}

Waist-to-height ratio: Waist circumference: BMI: Central obesity: Abdominal obesity: Obesity

\section{Introduction}

A variety of anthropometric indices have been used as a proxy for total fat or abdominal fat to assess risk for diseases, particularly CVD and diabetes. The most widely recognised is the BMI. Although this measure is correlated with total body fat, it does not distinguish fat from muscle or between different body fat distributions ${ }^{(1)}$. In the mid 20th century it was first observed that individuals with a central fat distribution were at greater health risk than those with peripheral fat ${ }^{(2,3)}$. Vague observed that individuals with a 'central type' of fat distribution (android shape) were at greater health risk than those whose fat was deposited 'peripherally' (gynoid shape).

It has only been accepted in the last two decades that health risks (predominantly CVD and diabetes) can be determined as much by the relative distribution of the excess fat as by its total amount. The use of imaging techniques such as computed tomography $(\mathrm{CT})^{(4)}$ and $\mathrm{MRI}^{(1,5)}$ indicated that the 'unhealthy apple shape' is associated with a preferential deposition of fat in the internal, visceral fat depots rather than the external, subcutaneous fat depots, this fat distribution being characteristic of the more 'healthy pear shape'(4).

An attempt to assess relative fat distribution was made with the ratio of waist circumference (WC) to hip circumference. This was shown to be a good predictor of health risk and was popular for many years ${ }^{(6)}$. However, although very useful for risk assessment, waist-to-hip ratio is not helpful in practical risk management because both waist and hip can decrease with weight reduction and so the ratio sometimes changes very little.

It was not until the end of the last century (1995) that WC by itself was proposed as an alternative proxy for central or abdominal obesity ${ }^{(7)}$. WC is strongly correlated with abdominal fat measures from advanced imaging techniques, and thought to represent fat stored in visceral depots.

Abbreviations: AUROC, area under receiver operator characteristics; DBP, diastolic blood pressure; HR, hazards ratio; ROC, receiver operator characteristics; RR, relative risk; SBP, systolic blood pressure; TC, total cholesterol; WC, waist circumference; WHtR, waist-toheight ratio.

* Corresponding author: Dr Margaret Ashwell OBE, email margaret@ashwell.uk.com 
However, WC may over- and under-evaluate risk for tall and short individuals with similar WC.

At about the same time, several researchers independently proposed the waist-to-height ratio (WHtR) as another proxy for central obesity, correcting the $\mathrm{WC}$ for the height of the individual ${ }^{(8-12)}$. Similar to WC, WHtR has been strongly correlated with abdominal fat measured using imaging techniques ${ }^{(1,13)}$

If an anthropometric index is to be used in a public health context and be used for screening, it is invariably useful to invoke cut-off or boundary values. The correction of WC for height offers the advantage that it is possible that a single WHtR boundary value may be useful in different ethnic, age and sex groups ${ }^{(14)}$, while WC requires population-specific boundary values ${ }^{(15)}$.

The present paper systematically reviews the evidence supporting the use of $\mathrm{WHtR}$, a proxy for abdominal fatness, as a predictor of CVD and diabetes, and their risk factors. In order to put the relationships into context, the review draws on evidence from prospective and cross-sectional studies, in adults and in children, which report relationships between WHtR and either BMI or WC, or both. Receiver operator characteristic (ROC) analyses are also summarised to indicate sensitivity and specificity of the potential predictors and to investigate possible boundary values for WHtR.

\section{Methods \\ Search methods}

A systematic literature search of electronic databases was conducted using Medline (from 1950 to week 3 of November 2008) and EMBASE (from 1980 until week 50 of 2008). Search terms were:

BMI OR body mass index OR body mass OR waist circumference OR wst circumference $\mathrm{OR}$ wst $\mathrm{OR}$ wst circum OR WC

\section{AND}

Waist-to-height OR waist to height OR waist height OR waist to ht OR waist-to-ht OR waist ht $\mathrm{OR}$ wst height OR wst ht OR WHtR OR waist circumference to height OR (stature AND girth).

Searches yielded 174203 articles on BMI, 13646 on WC and 286 on WHtR. Combining categories (to select papers including WHtR and either BMI or WC) and removing duplicates yielded 156 articles. All additional searches were completed up to 19 April 2009. This resulted in some 2009 publications being included. An additional twenty papers were found by hand-searching bibliographies of identified publications and key obesity journals for new or advance online publications and an additional search for waiststature papers. Where there was difficulty locating the full-text versions of articles and in extracting suitable information, authors were asked to supply. Two reviewers (L. M. B. and M. A.) assessed the suitability of these 176 articles, independently, using the following inclusion and exclusion criteria.

Study inclusion criteria. Inclusion criteria were: (1) human subjects, male, female or mixed, any age, adults or children, any ethnic group; (2) primary studies, either prospective or cross-sectional design; (3) WHtR and either BMI or WC measured at least once; (4) studies must also have a mortality, a cardiometabolic disease endpoint or cardiometabolic risk outcome measure, and present the relationship between obesity and the disease endpoint or risk outcome.

Study exclusion criteria. Exclusion criteria were: (1) literature reviews, intervention studies (although include and use baseline data if they fit inclusion criteria); (2) papers not written in English.

\section{Search results}

Figure 1 shows the selection of articles for inclusion in the present review. Of the 176 'database' identified articles, screening papers by title and abstract identified sixty-three as unsuitable. Reviewing the remaining papers from the full text identified a further thirty-seven as unsuitable. This gave a total of ninety-eight papers to be excluded from the review, for the following reasons: eight papers were written in a foreign language, forty-eight did not contain a suitable metabolic outcome measure, seven did not present results for WHtR and either BMI or WC, three were review articles, three were meta-analyses of previously published data, three were intervention studies, two were published letters and twenty did not contain suitable statistical analysis. Finally, we were unable to obtain full-text copies of four publications ${ }^{(16-19)}$.

A total of seventy-eight papers met the inclusion criteria for the systematic review.

\section{Results}

The results have been divided by study design (prospective or cross-sectional) and subdivided by health outcome, diabetes and CVD including their respective traditional risk factors. Studies in children have been treated separately. Finally, separate to the analyses of all the data in the systematic review, we present the results of those studies found in the systematic review that included ROC analysis. Significance of study results is as reported in each article; in all cases this is $P<0 \cdot 05$.

\section{Prospective studies in adults}

There were twenty-two separate publications that analysed prospective datasets in adults.

Diabetes outcomes. Table 1 includes the details of nine prospective studies with diabetes as the outcome measure ${ }^{(20-28)}$. However, two publications described populations derived from the same study group, with the more recent paper describing a smaller subpopulation ${ }^{(20,27)}$.

Table 2 shows the predictive power of WHtR, WC and BMI for diabetes outcomes. Significant predictors were defined as significantly increased OR or hazards ratios (HR) of developing diabetes. WHtR and WC were significant predictors in prospective analyses within six of nine studies, which included men ${ }^{(22)}$, men and women ${ }^{(24,25,27,28)}$ and in men and women of different ethnic backgrounds ${ }^{(23)}$. 


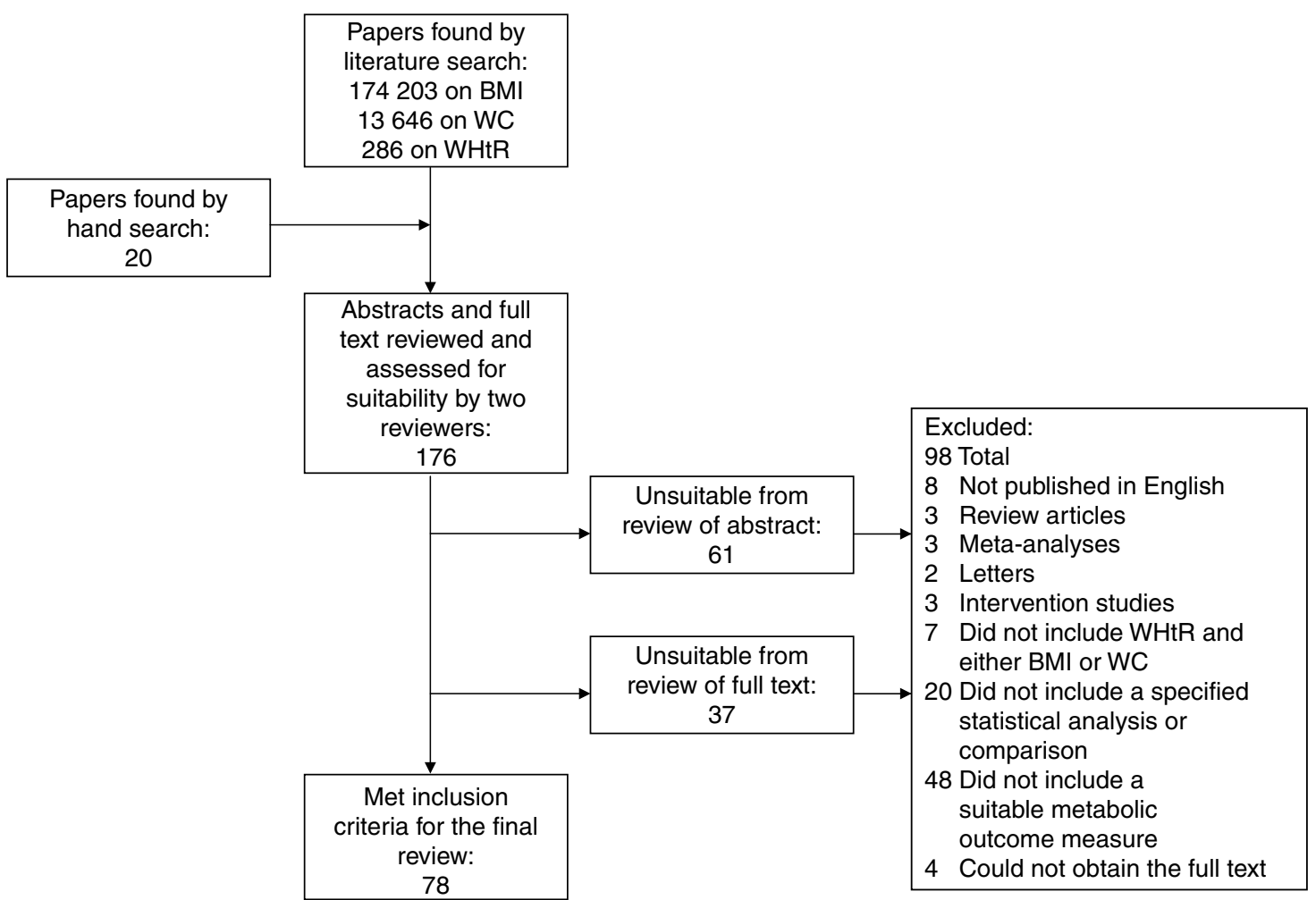

Fig. 1. Flow of the systematic review procedure. WC, waist circumference; WHtR, waist-to-height ratio.

BMI was a significant predictor in five of these same nine studies. Hadaegh et al. ${ }^{22)}$ showed no significant increase in risk across four BMI groups in risk of developing diabetes, after adjustment for additional factors, whilst for WC and WHtR groups there was a significant increased risk.

Other studies were significant in some, but not all of the analyses. Chei et al. ${ }^{(21)}$ and Sargeant et al. ${ }^{(26)}$ showed that WHtR, WC and BMI were significant predictors in women, but not men. Bray et al. ${ }^{(20)}$ showed that WHtR, WC and BMI were significant in men, but not women. This contrasted with the result in the larger group from the same population ${ }^{(27)}$, where WHtR, WC and BMI were significant predictors of diabetes in both men and women.

The values of OR and HR were similar for each of the three anthropometrical indices in most studies (values not presented in Table 2). However, in the Diabetes Prevention Program (DPP) population, the HR for WC in men was 1.42 $(95 \%$ CI $1.14,1.77)$ for a 1 SD increase, while for WHtR in men the HR was $1.32(95 \%$ CI 1.08, 1.63) and for BMI was $1.30(95 \% \text { CI } 1.07,1.57)^{(27)}$. Bray et al. reported similar trends in their subpopulation analysis of the $\mathrm{DPP}^{(20)}$. In contrast, each of the categories used by Hadaegh et al. gave higher relative risk (RR) for $\mathrm{WHtR}$ than $\mathrm{WC}$ or $\mathrm{BMI}^{(22)}$.

CVD outcomes. Table 1 includes the details of prospective studies with any aspect of CVD as an outcome. In total, fourteen populations were studied, in fifteen separate analyses. One publication described two separate analyses in two distinct populations, the Women's Health Study and the Physicians' Health Study, which are tabulated separately ${ }^{(29)}$. Two publications described the same study population, with different outcomes and durations of follow-up, and are tabulated separately ${ }^{(30,31)}$.

The studies have been subdivided by type of outcome, CVD events (fatal and non-fatal) and all-cause mortality $^{(29,31-37)}$, stroke ${ }^{(30,38)}$ and hypertension and blood pressure ${ }^{(21,39-41)}$. One study was analysed for both diabetes and hypertension (included twice in Table 1$)^{(21)}$, while one study $^{(35)}$ was included after the addition of data published subsequently in response to a letter to the editor ${ }^{(42)}$.

WHtR and WC were again significant predictors of outcomes in most (twelve of fifteen) of the studies, with BMI being a significant predictor in fewer (nine of fourteen) studies (Table 2). In cases where the anthropometric indices were not significant for the whole population, they were often significant in subpopulation analysis. In general, WC and WHtR tended to be significant more often than BMI. For example, Fuchs et al. found that WC and WHtR, but not BMI, were significant for hypertension in all study groups $^{(39)}$, and Wessel et al. found that WC and WHtR, but not BMI, were significant for all cardiovascular events, fatal and non-fatal ${ }^{(37)}$.

The values of the statistical ratios ( $O R$ and $\mathrm{HR}$ ) were similar for WHtR, WC and BMI in most studies (values not shown in Table 2). For example, Page et al. showed very similar OR after adjustment for multiple additional factors, 1.15 (95\% CI $1.10,1.21)$ for WHtR, 1.17 (95\% CI 1.11 , $1.25)$ for $\mathrm{WC}$ and $1.15(95 \%$ CI $1.07,1.24)$ for $\mathrm{BMI}^{(34)}$. In two studies WHtR was a stronger predictor than WC. Aekplakorn et al. showed an OR per $1 \mathrm{SD}$ increase in WHtR of $1.53(95 \%$ CI $1.21,1.95)$ and for WC of $1.35(95 \% \mathrm{CI}$ $1.06,1.72)^{(32)}$. Pischon et al. showed for WHtR a RR for 


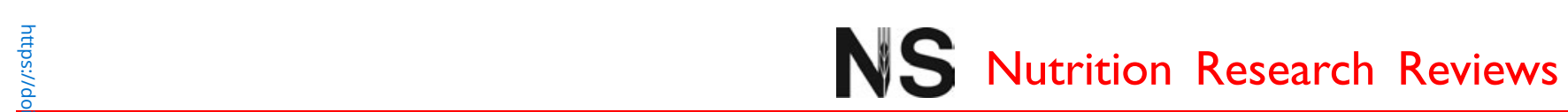

Table 1. Details of prospective studies in adults with diabetes or CVD as the outcome measure

\begin{tabular}{|c|c|c|c|c|c|c|c|c|}
\hline \multirow[b]{2}{*}{ Study reference } & \multicolumn{3}{|c|}{ Study design } & \multicolumn{5}{|c|}{ Population } \\
\hline & $\begin{array}{l}\text { Follow-up } \\
\text { duration (years) }\end{array}$ & Outcome & Analysis type & Subjects $(n)$ & Country & Age (years) & Sex & Inclusion criteria \\
\hline Bray et al. (2008) & 3.2 & Diabetes & HR per 1 sD increase & 108 & USA & $\geq 25$ & $\mathrm{M}$ and $\mathrm{F}$ & $\begin{array}{l}\text { Diabetes Prevention Program, } \\
\text { control group } \\
\text { BMI } \geq 24 \mathrm{~kg} / \mathrm{m}^{2}, \mathrm{IGT}\end{array}$ \\
\hline Chei et al. (2008) ${ }^{(21)}$ & 10.4 & Diabetes & OR per $1 \mathrm{sD}$ increase & 3391 & Japan & $40-69$ & $\begin{array}{l}\mathrm{M}(n 1102) \\
\mathrm{F}(n 2289)\end{array}$ & Non-diabetic, non-hypertensive \\
\hline Hadaegh et al. (2006) ${ }^{(22)}$ & 3.6 & Diabetes & $\begin{array}{l}\text { RR per categorical } \\
\text { increase }\end{array}$ & 1852 & Iran & $\geq 20$ & $M$ & $\begin{array}{l}\text { Tehran Lipid and Glucose Study } \\
\text { Non-diabetic }\end{array}$ \\
\hline Mansour \& Al-Jazairi (2007) ${ }^{(24)}$ & 5 & Diabetes & $\begin{array}{l}\text { Comparison of means } \\
\text { in diabetics } \\
v \cdot \text { non-diabetics }\end{array}$ & 13730 & Iraq & $\geq 18$ & $\begin{array}{l}\mathrm{M}(n \mathrm{n} 7101) \\
\mathrm{F}(n 6629)\end{array}$ & Non-diabetic \\
\hline MacKay et al. (2009) ${ }^{(23)}$ & 5.2 & Diabetes & OR per 1 SD increase & 1073 & USA & $40-69$ & $\begin{array}{l}\mathrm{M}(n \text { 472); } \\
\mathrm{F}(n \text { 601) }\end{array}$ & $\begin{array}{l}\text { Insulin Resistance Atherosclerosis } \\
\text { Study Non-Hispanic White } \\
(n 430) \text {, African-American } \\
\text { (n 282), Hispanic (n 361) }\end{array}$ \\
\hline Nyamdorj et al. (2009) & 5,6 and 11 & Diabetes & HR per 1 SD increase & 3945 & Mauritius & $25-74$ & $\begin{array}{l}\mathrm{M}(n-1841) \\
\mathrm{F}(n 2104)\end{array}$ & $\begin{array}{l}\text { Non-diabetic, no hypertension, } \\
\text { CVD or gout }\end{array}$ \\
\hline Sargeant et al. (2002) & 4 & Diabetes & OR per unit increase & 728 & Jamaica & $25-74$ & M (n 290); & Non-diabetic \\
\hline Anonymous $(2006)^{(27)}$ & 3.2 & Diabetes & HR per 1 sD increase & 1070 & USA & $\geq 25$ & $\begin{array}{l}\mathrm{M}(n 332) \\
\quad \mathrm{F}(n 738)\end{array}$ & $\begin{array}{l}\text { Diabetes Prevention Program, } \\
\text { control group BMI } \geq 24 \mathrm{~kg} / \mathrm{m}^{2}, \\
\text { IGT, and IFG }(5.3-6.9 \mathrm{~mm} / /) \text {. }\end{array}$ \\
\hline Tulloch-Reid et al. (2003) & 5.25 & Diabetes & HR per 1 sD increase & 1614 & USA & $\geq 18$ & $\begin{array}{l}\mathrm{M}(n 624) \\
\mathrm{F}(n 990)\end{array}$ & $\begin{array}{l}\text { Pima Indian population } \\
\text { Non-pregnant, non-diabetic }\end{array}$ \\
\hline Aekplakorn et al. $(2007)^{(32)}$ & 17 & $\begin{array}{l}\text { CVD (fatal and } \\
\text { non-fatal MI) }\end{array}$ & HR per 1 sD increase & 2536 & Thailand & $35-59$ & M & \\
\hline Cox et al. $(1998)^{(33)}$ & 7 & $\begin{array}{l}\text { CVD morbidity } \\
\text { and mortality }\end{array}$ & OR per quintile & 2854 & UK & $35-75$ & $\begin{array}{l}\mathrm{M}(n 1284) \\
\mathrm{F}(n 1570)\end{array}$ & $\begin{array}{l}\text { Health and Lifestyle Survey } \\
\text { No known diabetes, cancer or CVD }\end{array}$ \\
\hline Gelber et al. (2008) ${ }^{(29)}$ & 9 & CVD events & $\begin{array}{l}\text { RR per categorical } \\
\text { increase }\end{array}$ & 16332 & USA & $40-84$ & M & Physicians' Health Study \\
\hline Gelber et al. $(2008)^{(29)}$ & 6 & CVD events & $\begin{array}{l}\text { RR per categorical } \\
\text { increase }\end{array}$ & 32700 & USA & $>45$ & $\mathrm{~F}$ & Women's Health Study \\
\hline Page et al. $(2009)^{(34)}$ & 16 & $\begin{array}{l}\text { CVD (fatal or } \\
\text { non-fatal MI) }\end{array}$ & HR per unit increase & 45563 & USA & $40-65$ & $\mathrm{~F}$ & $\begin{array}{l}\text { Nurses' Health Study } \\
\text { No cancer, heart disease or stroke }\end{array}$ \\
\hline $\begin{array}{l}\text { Pischon et al. }(2008)^{(35)} \text { with } \\
\text { additional data in comment } \\
\text { Gaglione et al. }(2009)^{(42)}\end{array}$ & 9.7 & Death (all causes) & RR per quintile & 359387 & $\begin{array}{l}\text { Nine European } \\
\text { countries }\end{array}$ & $25-70$ & $\begin{array}{l}\mathrm{M}(34.6 \%) \\
\mathrm{F}(65.4 \%)\end{array}$ & $\begin{array}{l}\text { European Prospective Investigation } \\
\text { into Cancer and Nutrition } \\
\text { No history of cancer, heart } \\
\text { disease or stroke }\end{array}$ \\
\hline $\begin{array}{l}\text { Welborn \& Dhaliwal } \\
(2007)^{(36)}\end{array}$ & 11 & $\begin{array}{l}\text { Death (all-cause } \\
\text { and CVD) }\end{array}$ & HR per 1 SD increase & 9309 & Australia & $20-69$ & $\begin{array}{l}\mathrm{M}(n 4508) \\
\quad \mathrm{F}(n 4698)\end{array}$ & \\
\hline Wessel et al. (2004) ${ }^{(37)}$ & 3.9 & $\begin{array}{l}\text { CVD events (fatal } \\
\text { and non-fatal) }\end{array}$ & $\begin{array}{l}\text { OR per unit increase } \\
\text { HR per unit increase }\end{array}$ & 906 & USA & Adult & $\mathrm{F}(1+400)$ & $\begin{array}{l}\text { Women's Ischemia Syndrome } \\
\text { Evaluation Study } \\
\text { Recruited with chest discomfort } \\
\text { or suspected MI }\end{array}$ \\
\hline Zhang et al. (2004) & 2.5 & $\begin{array}{l}\text { CVD (fatal or } \\
\text { non-fatal MI) }\end{array}$ & RR per tertile increase & 67334 & China & $40-70$ & $\mathrm{~F}$ & $\begin{array}{l}\text { Shanghai Women's Health Study } \\
\text { No history of stroke, CVD or cancer }\end{array}$ \\
\hline Lu et al. $(2006)^{(38)}$ & 11 & All stroke & OR per quintile & 33578 & Sweden & $30-50$ & $\mathrm{~F}$ & $\begin{array}{l}\text { Swedish Women's Lifestyle and } \\
\text { Health Cohort Study }\end{array}$ \\
\hline Zhang et al. $(2009)^{(30)}$ & $7 \cdot 3$ & Total stroke & HR per quintile & 74942 & China & $40-70$ & $\mathrm{~F}$ & Shanghai Women's Health Study \\
\hline Chei et al. $(2008)^{(21)}$ & $10 \cdot 4$ & Hypertension & OR per 1 sD increase & 2972 & Japan & $40-69$ & $\begin{array}{l}\mathrm{M}(n-974) \\
\mathrm{F}(n 1998)\end{array}$ & No hypertension \\
\hline Fuchs et al. $(2005)^{(39)}$ & 5.6 & Hypertension & HR per unit increase & 592 & Brazil & $18-80$ & $\begin{array}{l}\mathrm{M}(n 255) \\
\mathrm{F}(n 337)\end{array}$ & No hypertension \\
\hline Nyamdorj et al. $(2008)^{(40)}$ & 5,6 and 11 & Hypertension & HR per 1 sD increase & 3634 & Mauritius & $25-74$ & $\begin{array}{l}\mathrm{M}(n 1658) \\
\mathrm{F}(n 1976)\end{array}$ & $\begin{array}{l}\text { Non-diabetic, no hypertension, } \\
\text { CVD or gout }\end{array}$ \\
\hline Panagiotakos et al. (2009) ${ }^{(41)}$ & 5 & Hypertension & HR per unit increase & 3042 & Mauritius & $>18$ & $\begin{array}{l}\mathrm{M}(n 1514) \\
\mathrm{F}(n 1528)\end{array}$ & No CVD \\
\hline
\end{tabular}

HR, hazards ratio; M, male; F, female; IGT, impaired glucose tolerance; IFG, impaired fasting glycaemia; RR, relative risk; MI, myocardial infarction. 
Table 2. Summary of the results of all prospective studies in adults, by outcome

\begin{tabular}{|c|c|c|c|c|c|c|c|c|c|}
\hline \multirow[b]{2}{*}{ Outcome } & \multicolumn{3}{|c|}{ WHtR } & \multicolumn{3}{|c|}{ WC } & \multicolumn{3}{|c|}{ BMI } \\
\hline & $\begin{array}{l}\text { Summary } \\
\text { score }^{\star}\end{array}$ & $\begin{array}{l}\text { Significant } \\
\text { in all analyses }\end{array}$ & $\begin{array}{l}\text { Significant in } \\
\text { some or none } \\
\text { of the analyses }\end{array}$ & $\begin{array}{l}\text { Summary } \\
\text { score }^{\star}\end{array}$ & $\begin{array}{l}\text { Significant } \\
\text { in all analyses }\end{array}$ & $\begin{array}{l}\text { Significant in } \\
\text { some or none } \\
\text { of the analyses }\end{array}$ & $\begin{array}{l}\text { Summary } \\
\text { score }^{\star}\end{array}$ & $\begin{array}{l}\text { Significant } \\
\text { in all analyses }\end{array}$ & $\begin{array}{l}\text { Significant } \\
\text { in some or none } \\
\text { of the analyses }\end{array}$ \\
\hline Diabetes & $6 / 9$ & $\begin{array}{l}\text { In men }{ }^{(22)} \text { men and } \\
\text { women } \\
\text { and men and } \\
\text { women of } \\
\text { different ethnic } \\
\text { backgrounds } \\
\end{array}$ & $\begin{array}{l}\text { In men, but } \\
\text { not women }{ }^{(20)} \text {, } \\
\text { in women, but } \\
\text { not men (21), } \\
\text { in whole population } \\
\text { and men } \\
\text { when analysed } \\
\text { separately, } \\
\text { but not women } \\
\text { separately } \\
\text { (26) }\end{array}$ & $6 / 9$ & $\begin{array}{l}\text { In men } \text { menen }^{(22)} \text { men and } \\
\text { women }(24,25,27,28) \\
\text { and men and } \\
\text { women of } \\
\text { different ethnic } \\
\text { backgrounds } \\
\end{array}$ & $\begin{array}{l}\text { In men, but not } \\
\text { women }{ }^{(20)} \text {, in } \\
\text { women, but not } \\
\text { men } n^{(21)} \text {, in whole } \\
\text { population and } \\
\text { men when analysed } \\
\text { separately, but } \\
\text { not women } \\
\text { separately } \\
\text { (26) }\end{array}$ & $5 / 9$ & $\begin{array}{l}\text { In men and } \\
\text { womene }{ }^{(24,25,27,28)} \\
\text { and in men and } \\
\text { women of } \\
\text { different ethnic } \\
\text { backgrounds } \\
\end{array}$ & $\begin{array}{l}\text { In men, but not women }{ }^{(20)} \text {, } \\
\text { in women, but not } \\
\text { men (21), before, but } \\
\text { not after adjustment } \\
\text { for IFG/IGT }{ }^{(22)} \text { and } \\
\text { in whole population } \\
\text { and men when } \\
\text { analysed separately, } \\
\text { but not women } \\
\text { separately } \\
\text { se) }\end{array}$ \\
\hline $\begin{array}{l}\text { CVD events } \\
\text { (fatal and non-fatal) }\end{array}$ & $7 / 9$ & $\begin{array}{l}\text { In men } \text { meg, }^{(29,32,36)} \\
\text { women } \\
\text { and men } \\
\text { and women } \\
\text { and wom }\end{array}$ & $\begin{array}{l}\text { In non-hypertensive } \\
\text { men, but } \\
\text { not whole } \\
\text { population } \\
\text { for all adverse } \\
\text { events but not } \\
\text { for obstructive } \\
\text { CAD, major } \\
\text { adverse events } \\
\text { or all-cause } \\
\text { mortality }\end{array}$ & $7 / 9$ & $\begin{array}{l}\text { In } \operatorname{men}^{(29,32,36)} \\
\text { women } \\
\text { and men and }{ }^{(29,34,36)} \\
\text { women } \\
\text { (35) }\end{array}$ & $\begin{array}{l}\text { In non-hypertensive } \\
\text { men, but not } \\
\text { whole population } \\
\text { or women } n^{(33)} \text {, for } \\
\text { all adverse events } \\
\text { but not for obstructive } \\
\text { CAD, major adverse } \\
\text { events or all cause } \\
\text { mortality }{ }^{\left({ }^{(37)}\right.}\end{array}$ & $6 / 8$ & $\begin{array}{l}\text { In men } \text { memen }^{(29,32,36)} \text { and } \\
\text { wom,34,36) }\end{array}$ & $\begin{array}{l}\text { Not in whole } \\
\text { population or } \\
\text { non-hypertensive } \\
\text { men and women } \\
\text { for obstructive CAD, } \\
\text { all adverse events, } \\
\text { major adverse events } \\
\text { or all-cause mortality }{ }^{(37)}\end{array}$ \\
\hline All stroke & $2 / 2$ & $\begin{array}{l}\text { In womene( }{ }^{(38)} \text { and in } \\
\text { women before } \\
\text { and after adjustment } \\
\text { for BMI }{ }^{(30)}\end{array}$ & & $2 / 2$ & $\begin{array}{l}\text { In women }{ }^{(38)} \text { and } \\
\text { in women } \\
\text { before and } \\
\text { after } \\
\text { adjustment for } \\
\text { BMI }^{(30)}\end{array}$ & & $1 / 2$ & In women ${ }^{(30)}$ & $\begin{array}{l}\text { Before, but not after } \\
\text { adjustment for history } \\
\text { of hypertension } \\
\text { and diabetes } \\
\text { (38) }\end{array}$ \\
\hline $\begin{array}{l}\text { Hypertension, } \\
\text { SBP and DBP }\end{array}$ & $3 / 4$ & $\begin{array}{l}\text { In men and } \\
\text { women } \text { n }^{(39,41)} \\
\text { and men and } \\
\text { women of } \\
\text { different ethnic } \\
\text { backgrounds }{ }^{(40)}\end{array}$ & $\begin{array}{l}\text { In urban men } \\
\text { and rural women, } \\
\text { but not rural } \\
\text { men and } \\
\text { urban women }{ }^{(21)}\end{array}$ & $3 / 4$ & $\begin{array}{l}\text { In men and } \\
\text { womene }{ }^{(39,41)} \\
\text { and men and } \\
\text { women } \\
\text { of different ethnic } \\
\text { backgrounds }{ }^{(40)}\end{array}$ & $\begin{array}{l}\text { In urban men and } \\
\text { urban and rural } \\
\text { women, but not } \\
\text { rural men }{ }^{(21)}\end{array}$ & $2 / 4$ & $\begin{array}{l}\text { In men and } \\
\text { women } \text { n }^{(41)} \\
\text { and men and } \\
\text { women of } \\
\text { different ethnic } \\
\text { backgrounds }^{(40)}\end{array}$ & $\begin{array}{l}\text { Not in whole study } \\
\text { population } \\
\text { men and rural wo women, } \\
\text { but not rural men and } \\
\text { urban women } \\
\text { (21) }\end{array}$ \\
\hline
\end{tabular}

WHtR, waist-to-height ratio; WC, waist circumference; IFG, impaired fasting glycaemia; IGT, impaired glucose tolerance; CAD, coronary artery disease; SBP, systolic blood pressure; DBP, diastolic blood pressure. * Summary score indicates the number of studies in which all the published analysis showed that the obesity measure was significant in predicting the outcome, out of the total number of studies for this outcome. Data analysed as odds or hazards ratio by cut-
off or group of the specified obesity measure. 
quintile $1 v$. quintile 5 of $2 \cdot 22(95 \%$ CI $1.94,2 \cdot 55)$ for men and $2.03(95 \%$ CI $1.76,2.34)$ for women ${ }^{(42)}$, but for WC, a RR of 2.05 (95\% CI 1.80, 2.33) for men and $1.78(95 \% \mathrm{CI}$ $1 \cdot 56,2 \cdot 04)$ for $\operatorname{women}^{(35)}$.

Waist-to-height ratio and waist circumference as independent predictors of disease after adjustment or stratification for BMI. Three studies determined whether WHtR and WC were significant predictors of outcome after adjustment for BMI. Two studies ${ }^{(30,35)}$ showed that both WHtR and WC were significant predictors of stroke and all-cause mortality, respectively. However, in the Shanghai Women's Health Study, analysed for a different outcome ${ }^{(31)}$, neither WHtR nor WC was a significant predictor of fatal and non-fatal MI, after adjustment for BMI.

\section{Cross-sectional studies in adults}

Cross-sectional outcome measures considered in the present review are any of the risk factors related to CVD and diabetes: blood pressure, lipid outcomes and insulin indices. For adults these have been considered separately, but for children, they have been tabulated and discussed together.

A total of forty-four cross-sectional adult populations were identified, published in forty-three separate publications ${ }^{(9,10,43-83)}$. Of these forty-four papers, nineteen publications had calculated OR or similar $(43-59,64,69)$ (Table 3), twenty-five papers presented correlation analyses $(9,48,55,57-63,66-68,71,73-83)$, four presented linear regression $^{(9,10,54,70)}$, one random effects ${ }^{(72)}$ and one paper compared means $^{(65)}$ (Table 4).

The majority of studies explored the relationship between the anthropometric indices and a wide range of disease risk factors, namely blood pressure, lipids and insulin indices, as continuous variables. A few studies explored the relationship between disease endpoints and the anthropometric indices and/or explored the relationship between disease endpoints and groups separated by boundary values for anthropometric indices (diabetes ${ }^{(43,49,51,55,56,58,69)}$, coronary artery disease $^{(46,57)}$, hypertension $(44,45,47,48,50,54,55,58,59,64,69)$ and dyslipidaemia $\left.{ }^{(44,45,48,49,53,56,58,59)}\right)$.

Table 5 shows the results of the cross-sectional studies with OR or similar analysis (categorical outcomes), and Table 6 shows the results of the studies with correlation or linear regression analysis (continuous outcomes).

Diabetes outcomes. OR analysis (Table 5) showed that WHtR was significantly associated with diabetes outcomes in six of seven studies, WC in six of six studies and BMI in six of seven studies. Two studies showed significantly increased odds of having diabetes for each 1 SD increase in WHtR, WC and BMI in German and Australian men and women, respectively ${ }^{(56,58)}$. The values of the OR were similar for WHtR, WC and BMI in these studies. One study showed significant increased odds of having diabetes across quartiles of WHtR, WC and BMI in Indian men and women $^{(43)}$, but the odds were slightly stronger for WHtR than other anthropometric indices (quartile $4 v$. quartile 1 was 6.69 (95\% CI 5.25, 8.53) for WHtR, 5.88 (95\% CI 4.66, 7.42) for WC and 4.01 (95\% CI 3.23, 4.99) for BMI). Only one study showed variance in results between the three anthropometric indices: Iranian women with high WC had significantly greater odds of having diabetes, but those with high WHtR and BMI did not ${ }^{(49)}$.

Correlation studies of fasting insulin (Table 6) showed strong and significant correlations in seven of seven studies for WHtR, WC and BMI. Correlations were not so often significant for fasting glucose (significant in twelve of eighteen studies for WHtR and BMI and ten of sixteen studies for WC).

CVD outcomes. Two cross-sectional studies (Table 5) determined odds of CVD and showed that odds of disease were significantly higher for increases in WHtR, WC and $\mathrm{BMI}^{(46,57)}$, though BMI was not significant in men ${ }^{(57)}$.

Hypertension outcomes. Of the studies determining odds of hypertension (Table 5), nine of ten showed WHtR to be significant in all analyses, eight of nine studies showed WC to be significant and ten of ten showed BMI to be significant. The results of the studies were consistent and showed increased odds of hypertension in different age ${ }^{(47)}, \operatorname{sex}^{(54,59)}$ and ethnic groups ${ }^{(50,58)}$ for WHtR, WC and BMI. The many correlation studies (Table 6) gave results that were generally significant for all anthropometric indices, with similar coefficients for systolic blood pressure (SBP) and diastolic blood pressure (DBP) ${ }^{(47,55,66)}$.

Lipid outcomes. Studies determining effects on various lipid outcomes were consistent in their results across the three anthropometric indices. Odds of dyslipidaemia (Table 5) were significantly increased for WHtR in three of four studies, for WC in four of four studies, and for BMI in three of four studies. Significant increased odds of dyslipidaemia were seen per $1 \mathrm{SD}$ increase in WHtR, WC and BMI in men and women ${ }^{(56)}$ and in different ethnic groups ${ }^{(58)}$. Similarly, increased odds were shown across quartiles of WHtR, WC and BMI for women and WHtR and WC for men ${ }^{(53)}$. However, Esmailzadeh et al. showed no significant increased odds of dyslipidaemia for a high WHtR, although the analysis was significant for $\mathrm{WC}$ and $\mathrm{BMI}^{(49)}$.

Cross-sectional studies also determined odds of total cholesterol (TC) (two studies), TAG (three studies), LDL (two studies), HDL (three studies), and TC:HDL (two studies). Bertsias et al. showed similar results for WC and WHtR, with the odds being significant for LDL and TC:HDL in men and women and TC in men ${ }^{(45)}$. However, Azizi et al. showed no significant increased odds of any lipid outcome for WC, but increased odds of high TC, TAG and LDL with both WHtR and BMI ${ }^{(44)}$.

Many correlation studies (Table 6) determined relationships with TC (sixteen studies), TAG (twenty-one studies), LDL (ten studies), HDL (nineteen studies), TC:HDL (three studies), LDL:HDL (three studies) and TAG:HDL (one study). Results tended to show stronger correlations for all anthropometric indices with TAG and HDL; a larger proportion of studies showed non-significant relationships with TC and LDL.

Waist-to-height ratio and waist circumference as independent risk factors for disease after adjustment or stratification for BMI. In order to determine whether WC 


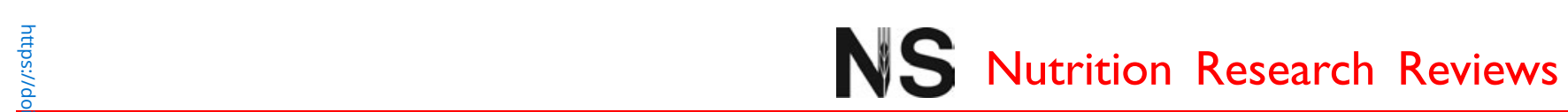

Table 3. Details of cross-sectional studies in adults: OR analysis

\begin{tabular}{|c|c|c|c|c|c|c|c|}
\hline \multirow[b]{2}{*}{ Study reference } & \multirow[b]{2}{*}{ Study design: analysis type } & \multirow[b]{2}{*}{ Outcomes } & \multicolumn{5}{|c|}{ Population } \\
\hline & & & Subjects $(n)$ & Country & Age (years) & Sex & Inclusion criteria \\
\hline Ajay et al. $(2008)^{(43)}$ & $\begin{array}{l}\text { OR, quartiles of WHtR, } \\
\text { WC and BMI }\end{array}$ & Diabetes & 10930 & India & $20-69$ & $\begin{array}{l}\mathrm{M}(n 6764) ; \\
\mathrm{F}(n 4166)\end{array}$ & $\begin{array}{l}\text { Industrial workers } \\
\text { and their families }\end{array}$ \\
\hline Azizi et al. $(2004)^{(44)}$ & $\begin{array}{l}\text { OR, cut-offs of WHtR, } \\
\text { WC and BMI }\end{array}$ & $\begin{array}{l}\text { Cut-offs for } \\
\text { hypertension, high TC, } \\
\text { TAG, LDL, HDL, TC:HDL }\end{array}$ & 9647 & Iran & $20-70$ & $\begin{array}{l}\mathrm{M}(n 3622) \\
\mathrm{F}(n 5025)\end{array}$ & \\
\hline Bertsias et al. $(2003)^{(45)}$ & $\begin{array}{l}\text { OR, cut-offs of WHtR, } \\
\text { WC and BMI }\end{array}$ & $\begin{array}{l}\text { Cut-offs for high SBP, DBP, FPG, } \\
\text { TC, TAG, HDL, LDL, TC:HDL }\end{array}$ & 989 & Greece & $20-40$ & $\begin{array}{l}\mathrm{M}(n 527) ; \\
\mathrm{F}(n 462)\end{array}$ & $\begin{array}{l}\text { Third year students, } \\
\text { University of Crete, } \\
\text { School of Medicine }\end{array}$ \\
\hline Brouwer et al. (2007) $)^{(46)}$ & $\begin{array}{l}\text { OR per } 1 \mathrm{SD} \text { increase } \\
\text { in WHtR, WC or BMI }\end{array}$ & CVD & 315 & The Netherlands & $18-80$ & $\begin{array}{l}\mathrm{M}(n \text { 225); } \\
\mathrm{F}(n \text { 90) }\end{array}$ & $\begin{array}{l}\text { SMART study } \\
\text { All have PAD: } \\
\text { n } 79 \text { CVD, } \\
\text { n } 236 \text { no CVD }\end{array}$ \\
\hline Cox et al. $(1997)^{(47)}$ & $\begin{array}{l}\text { OR, quintiles of WHtR, } \\
\text { WC and BMI }\end{array}$ & $\begin{array}{l}\text { Hypertension }(>140 / 90 \mathrm{mmHg} \\
\text { or treatment) }\end{array}$ & 5991 & UK & $\begin{array}{l}18-39 \text { (young) } \\
\text { and } 40-64 \text { (old) }\end{array}$ & $\begin{array}{l}\mathrm{M}(n-2712) \\
\mathrm{F}(n 3279)\end{array}$ & \\
\hline $\begin{array}{l}\text { Esmaillzadeh } \\
\text { et al. }(2004)^{(48)}\end{array}$ & $\begin{array}{l}\text { OR, cut-offs of WHtR, } \\
\text { WC and BMI }\end{array}$ & $\begin{array}{l}\text { One risk factor for hypertension, } \\
\text { diabetes or DYSLIP }\end{array}$ & 4449 & Iran & $18-74$ & $\mathrm{M}$ & \\
\hline $\begin{array}{l}\text { Esmaillzadeh } \\
\quad \text { et al. (2006) }\end{array}$ & $\begin{array}{l}\text { OR, cut-offs of WHtR, } \\
\text { WC and BMI }\end{array}$ & $\begin{array}{l}\text { Hypertension, diabetes, DYSLIP, } \\
\text { MetS one risk factor }\end{array}$ & 5073 & Iran & $18-74$ & $\mathrm{~F}$ & $\begin{array}{l}\text { No diabetes or } \\
\text { dyslipidaemia } \\
\text { medication }\end{array}$ \\
\hline $\begin{array}{l}\text { Ghosh \& } \\
\text { Bandyopadhyay } \\
(2007)^{(64)}\end{array}$ & $\begin{array}{l}\text { OR, unit increase in } \\
\text { WHtR, WC and BMI }\end{array}$ & Hypertension & 180 & India & $20-61$ & M & \\
\hline Harris et al. $(2000)^{(50)}$ & $\begin{array}{l}\text { OR, quintiles of WHtR, } \\
\text { WC and BMI }\end{array}$ & Hypertension & 15063 & USA & $45-64$ & $\begin{array}{l}\mathrm{M}(n 5332+1523) \\
\mathrm{F}(n 5766+2442)\end{array}$ & ARIC study \\
\hline He et al. $(2008)^{(51)}$ & $\begin{array}{l}\text { Prevalence ratio, } \\
\text { above and below a } \\
\text { cut-off of WHtR, } \\
\text { WC and BMI }\end{array}$ & $\begin{array}{l}\text { Glucose tolerance abnormalities } \\
\text { (T2D, IFG or IGT) }\end{array}$ & 50905 & China & $18-79$ & $\begin{array}{l}\mathrm{M}(n 23980) ; \\
\mathrm{F}(n 26925)\end{array}$ & $\begin{array}{l}\text { China National } \\
\text { Nutrition and } \\
\text { Health Survey }\end{array}$ \\
\hline Hsieh et al. $(2000)^{(52)}$ & $\begin{array}{l}\text { OR, split into groups } \\
\text { by BMI and WHtR } \\
\text { combined }\end{array}$ & Various metabolic risk factors & 2668 & Japan & $21-85$ & M & \\
\hline Jeong et al. $(2005)^{(53)}$ & $\begin{array}{l}\text { OR, quartiles of WHtR, } \\
\text { WC and BMI }\end{array}$ & DYSLIP & 1032 & Korea & $\geq 50$ & $\begin{array}{l}\mathrm{M}(n 356) \\
\mathrm{F}(n 676)\end{array}$ & $\begin{array}{l}\text { History of stroke } \\
\text { or CVD excluded }\end{array}$ \\
\hline Kaur et al. $(2008)^{(69)}$ & $\begin{array}{l}\text { OR, quintiles of WHtR, } \\
\text { WC and BMI }\end{array}$ & Hypertension and diabetes & 2148 & India & $18-69$ & M & \\
\hline Sakurai et al. $(2006)^{(54)}$ & $\begin{array}{l}\text { Rate ratio per } 1 \mathrm{SD} \\
\text { increase in WHtR, } \\
\text { WC or BMI }\end{array}$ & Hypertension & 4557 & Japan & $35-59$ & $\begin{array}{l}\mathrm{M}(n 2935) \\
\mathrm{F}(n 1622)\end{array}$ & \\
\hline Sayeed et al. $(2003)^{(55)}$ & $\begin{array}{l}\text { OR, quartiles of WHtR, } \\
\text { WC and BMI }\end{array}$ & $\begin{array}{l}\text { Diabetes, IFG and systolic } \\
\text { hypertension }\end{array}$ & 1531 & Bangladesh & $\geq 20$ & $\mathrm{M}$ and $\mathrm{F}$ & \\
\hline Schneider et al. $(2007)^{(56)}$ & $\begin{array}{l}\text { OR, per } 1 \text { SD increase } \\
\text { in WHtR, WC and BMI }\end{array}$ & Diabetes, MetS and DYSLIP & 5377 & Germany & $20-79$ & $\begin{array}{l}\mathrm{M}(n 2016) \\
\mathrm{F}(n 3361)\end{array}$ & $\begin{array}{l}\text { DETECT Without } \\
\text { arteriosclerotic } \\
\text { disease }\end{array}$ \\
\hline Tseng $(2008)^{(57)}$ & $\begin{array}{l}\text { OR per } 1 \mathrm{sD} \text { increase } \\
\text { in } \mathrm{WH} \mathrm{HR}, \mathrm{WC} \text { and } \mathrm{BMI}\end{array}$ & CVD & 1345 & Taiwan & $\geq 18$ & $\begin{array}{l}\mathrm{M}(n 646) \\
\mathrm{F}(n 699)\end{array}$ & $\begin{array}{l}\text { Inclusion of type } 1 \\
\text { and } 2 \text { diabetics }\end{array}$ \\
\hline Wang et al. $(2007)^{(58)}$ & $\begin{array}{l}\text { OR per } 1 \mathrm{SD} \text { increase } \\
\text { in WHtR, WC or BMI } \\
\text { Correlation }\end{array}$ & $\begin{array}{l}\text { Diabetes, hypertension, DYSLIP } \\
\text { SBP, DBP, glucose, TC, } \\
\text { LDL, HDL, TAG }\end{array}$ & 1186 & Australia & Adult & $\mathrm{M}$ and $\mathrm{F}$ & $\begin{array}{l}\text { Aboriginal }(n 747) \\
\text { or Torres Strait } \\
\text { Islanders }(n 439)\end{array}$ \\
\hline Wu et al. $(2007)^{(59)}$ & $\begin{array}{l}\text { OR, cut-off for WHtR, } \\
\text { WC and BMI } \\
\text { Correlation }\end{array}$ & $\begin{array}{l}\text { Hypertension, high TAG, low HDL } \\
\text { SBP, DBP, TAG, HDL, HbA1c, } \\
\text { FPG }\end{array}$ & 411 & China & $\geq 40$ & $\begin{array}{l}\mathrm{M}(n 198) \\
\mathrm{F}(n 213)\end{array}$ & $\begin{array}{l}\text { Newly diagnosed } \\
\text { type } 2 \text { diabetes, } \\
\text { no history of } \\
\text { diabetes or diabetic } \\
\text { complications }\end{array}$ \\
\hline
\end{tabular}

WHtR, waist-to-height ratio; WC, waist circumference; M, male; F, female; TC, total cholesterol; SBP, systolic blood pressure; DBP, diastolic blood pressure; FPG, fasting plasma glucose; SMART, Second Manifestations

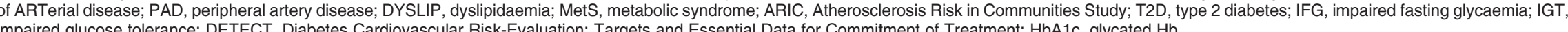

impaired glucose tolerance; DETECT, Diabetes Cardiovascular Risk-Evaluation: Targets and Essential Data for Commitment of Treatment; HbA1c, glycated Hb. 


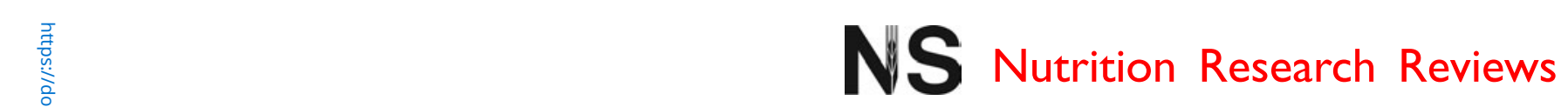

Table 4. Details of cross-sectional studies in adults: correlation analysis

\begin{tabular}{|c|c|c|c|c|c|c|c|}
\hline \multirow[b]{2}{*}{ Study reference } & \multirow[b]{2}{*}{$\begin{array}{l}\text { Study design: } \\
\text { analysis type }\end{array}$} & \multirow[b]{2}{*}{ Outcomes } & \multicolumn{5}{|c|}{ Population } \\
\hline & & & Subjects $(n)$ & Country & Age (years) & Sex & Inclusion criteria \\
\hline $\begin{array}{l}\text { Bosy-Westphal } \\
\text { et al. }(2006)^{(60)}\end{array}$ & Correlation & $\begin{array}{l}\text { SBP, TC, TAG, } \\
\text { HDL, HOMA-IR }\end{array}$ & 335 & Germany & $28-84$ & $\begin{array}{l}\mathrm{M}(n 144) \\
\mathrm{F}(n 191)\end{array}$ & $\begin{array}{l}\text { KOPS } \\
\text { Three generations including } \\
\text { one with overweight or obesity }\end{array}$ \\
\hline $\begin{array}{l}\text { Can et al. } \\
(2009)^{(61)}\end{array}$ & Correlation & $\begin{array}{l}\text { SBP, FPG, HOMA-IR, } \\
\text { TC, TAG, LDL, HDL }\end{array}$ & 1692 & Turkey & $\geq 18$ & $\begin{array}{l}\mathrm{M}(n 571) \\
\mathrm{F}(n 1121)\end{array}$ & \\
\hline $\begin{array}{l}\text { Chehrei } \\
\text { et al. }(2007)^{(62)}\end{array}$ & Correlation & $\begin{array}{l}\text { FPG, TC, TAG, LDL, } \\
\text { HDL, TC:HDL, LDL:HDL }\end{array}$ & 750 & Iran & $\begin{array}{l}\text { Mean } 43 \cdot 6 \\
\text { Mean } 40.4\end{array}$ & $\begin{array}{l}\mathrm{M}(n 170) \\
\mathrm{F}(n 580)\end{array}$ & $\begin{array}{l}\text { Excluded hypertension and } \\
\text { diabetes }\end{array}$ \\
\hline $\begin{array}{l}\text { Deshmukh } \\
\text { et al. }(2006)^{(63)}\end{array}$ & Correlation & SBP, DBP & 2700 & India & $\geq 18$ & $\begin{array}{l}\mathrm{M}(n 1059) \\
\mathrm{F}(n 1641)\end{array}$ & Household recruitment \\
\hline $\begin{array}{l}\text { Esmaillzadeh } \\
\text { et al. }(2004)^{(48)}\end{array}$ & Correlation & $\begin{array}{l}\text { SBP, DBP, FPG, TC, } \\
\text { TAG, HDL, LDL }\end{array}$ & 4449 & Iran & $18-74$ & M & \\
\hline $\begin{array}{l}\text { Ho et al. } \\
(2003)^{(66)}\end{array}$ & Correlation & $\begin{array}{l}\text { SBP, DBP, FPI, FPG, } \\
\text { 2hrG, TC, TAG, } \\
\text { LDL, HDL }\end{array}$ & 2895 & Hong Kong & $25-74$ & $\begin{array}{l}\mathrm{M}(n 1412) \\
\mathrm{F}(n 1483)\end{array}$ & $\begin{array}{l}\text { Exclusion of all with serious } \\
\text { diseases, for example, } \\
\text { cancer or hospitalised } \\
\text { subjects }\end{array}$ \\
\hline $\begin{array}{l}\text { Hsieh } \\
\text { et al. }(2003)^{(67)}\end{array}$ & Correlation & MetS score & 8278 & Japan & $\begin{array}{l}\text { Mean } 49.5 \\
\text { Mean } 51.9\end{array}$ & $\begin{array}{l}\mathrm{M}(n 6141) \\
\mathrm{F}(n 2137)\end{array}$ & \\
\hline $\begin{array}{l}\text { Hsieh \& } \\
\text { Muto (2005) }\end{array}$ & Correlation & $\begin{array}{l}\text { Sum of coronary } \\
\text { risk factors }\end{array}$ & 6521 & Japan & Adult & $\begin{array}{l}\mathrm{M}(n \text { 4668); } \\
\mathrm{F}(n 1853)\end{array}$ & $\mathrm{BMI}<25 \mathrm{~kg} / \mathrm{m}^{2}$ \\
\hline $\begin{array}{l}\text { Kotchen } \\
\text { et al. }(2008)^{(71)}\end{array}$ & Correlation & SBP, DBP & 2747 & $\begin{array}{l}\text { USA, } \\
\text { African- } \\
\text { American }\end{array}$ & $18-55$ & $\mathrm{M}$ and $\mathrm{F}$ & $\begin{array}{l}\mathrm{BMI}<36 \mathrm{~kg} / \mathrm{m}^{2}, \text { non-diabetic, } \\
\text { non-pregnant }\end{array}$ \\
\hline $\begin{array}{l}\text { Kotchen } \\
\text { et al. }(2008)^{(71)}\end{array}$ & Correlation & SBP, DBP & 3090 & USA & $18-55$ & $\mathrm{M}$ and $\mathrm{F}$ & $\begin{array}{l}\text { NHANES } \\
\text { Non-anti-hypertensive } \\
\text { treatment, non-pregnant }\end{array}$ \\
\hline $\begin{array}{l}\text { Lopatynski } \\
\text { et al. }(2003)^{(73)}\end{array}$ & Correlation & $\mathrm{FPG}$ and $2 \mathrm{hrG}$ & 1965 & Poland & $\geq 35$ & $M$ and $F$ & \\
\hline $\begin{array}{l}\text { Lovegrove } \\
\quad \text { et al. }(2002)^{(74)}\end{array}$ & Correlation & $\begin{array}{l}\text { FPI, FPG, TC, } \\
\text { TAG, LDL, HDL }\end{array}$ & 28 & UK & Adult & $\mathrm{F}$ & $\begin{array}{l}\text { HRT, } \mathrm{BMI}<18 \text { or }>37 \mathrm{~kg} / \mathrm{m}^{2} \\
\text { age }>80 \text { years excluded }\end{array}$ \\
\hline $\begin{array}{l}\text { Maher } \\
\text { et al. }(2009)^{(75)}\end{array}$ & Correlation & $\begin{array}{l}\text { SBP, DBP, HOMA-IR, } \\
\text { HbA1c, FPG, FPI, } \\
\text { TC, TAG, LDL, HDL }\end{array}$ & 100 & Ireland & $\geq 18$ & $\begin{array}{l}\mathrm{M}(n 29) \\
\mathrm{F}(n 71)\end{array}$ & $\begin{array}{l}\text { Inclusion of never smokers, } \\
\text { no history of vascular events, } \\
\text { no hypertension, T2D } \\
\text { or familial hyperchole- } \\
\text { sterolaemia }\end{array}$ \\
\hline $\begin{array}{l}\text { Mukuddem-Petersen } \\
\text { et al. }(2006)^{(76)}\end{array}$ & Correlation & $\begin{array}{l}\text { SBP, DBP, FPG, } 2 \mathrm{hrG} \text {, } \\
\mathrm{HbA1c} \text {, TAG, HDL }\end{array}$ & 826 & Holland & $56-83$ & $\begin{array}{l}\mathrm{M}(n \text { 389); } \\
\mathrm{F}(n \text { 437) }\end{array}$ & $\begin{array}{l}\text { Hoorn Study } \\
\text { Excluded if taking lipid-lowering, } \\
\text { anti-hypertensive or } \\
\text { anti-diabetic medication }\end{array}$ \\
\hline $\begin{array}{l}\text { Paniagua et al. } \\
(2008)^{(77)}\end{array}$ & Correlation & $\begin{array}{l}\text { SBP, DBP, FPG, } \\
\text { TAG, HDL }\end{array}$ & 1391 & Thailand & $\geq 35$ & $\begin{array}{l}\mathrm{M}(n \text { 451); } \\
\mathrm{F}(n 940)\end{array}$ & $\begin{array}{l}\text { Excluded if taking anti-diabetic, } \\
\text { anti-hypertensive or } \\
\text { lipid-lowering medication }\end{array}$ \\
\hline $\begin{array}{l}\text { Patel et al. } \\
(1999)^{(78)}\end{array}$ & Correlation & 2hrG, FPG, HDL & 1606 & UK & $25-64$ & $\mathrm{M}$ and $\mathrm{F}$ & $\begin{array}{l}\text { Chinese, European and } \\
\text { South Asian }\end{array}$ \\
\hline $\begin{array}{l}\text { Rissanen et al. } \\
\left(_{(1997)^{(79)}}\right.\end{array}$ & Correlation & $\begin{array}{l}\text { FPI, FPG, 2hrG, } \\
\text { TAG, LDL, HDL }\end{array}$ & 43 & Finland & $29-64$ & $\mathrm{~F}$ & $\begin{array}{l}\text { BMI } 28-42 \mathrm{~kg} / \mathrm{m}^{2} \\
\text { Excluded with diabetes, thyroid, } \\
\text { liver, kidney diseases or } \\
\mathrm{DBP} \geq 105 \mathrm{mmHg}\end{array}$ \\
\hline
\end{tabular}


WS Nutrition Research Reviews

\begin{tabular}{|c|c|c|c|c|c|c|c|}
\hline Sattar et al. $(1998)^{(80)}$ & Correlation & $\begin{array}{l}\text { MAP, FPI, FPG, TC, } \\
\text { TAG, LDL, HDL, } \\
\text { LDL:HDL }\end{array}$ & 191 & UK & $18-69$ & $\begin{array}{l}\mathrm{M}(n 93) \\
\mathrm{F}(n 98)\end{array}$ & Healthy \\
\hline Sayeed et al. (2003) ${ }^{(55)}$ & Correlation & $\begin{array}{l}\text { SBP, DBP, FPG, TC, } \\
\text { TAG, HDL:TAG, HDL:TC }\end{array}$ & 4923 & Bangladesh & $\geq 20$ & $\mathrm{M}$ and $\mathrm{F}$ & \\
\hline $\begin{array}{l}\text { Thomas } \\
(1999)^{(81)}\end{array}$ & Correlation & DBP & 50 & Hong Kong & $20-55$ & $\mathrm{M}$ and $\mathrm{F}$ & $\begin{array}{l}\text { Hypertensive (sibling pair } \\
\text { analysis not used) }\end{array}$ \\
\hline Tseng $(2008)^{(57)}$ & Correlation & $\begin{array}{l}\text { SBP, DBP, FPG, } \\
\text { HbA1c, TC, TAG }\end{array}$ & 1345 & Taiwan & $\geq 18$ & $\begin{array}{l}\mathrm{M}(n 646) \\
\mathrm{F}(n 699)\end{array}$ & $\begin{array}{l}\text { Inclusion of type } 1 \text { and } 2 \\
\text { diabetics }\end{array}$ \\
\hline $\begin{array}{l}\text { Turcato et al. } \\
\text { (2000) }^{(82)}\end{array}$ & Correlation & $\begin{array}{l}\text { SBP, DBP, FPG, 2hrG, } \\
\text { TC, TAG, HDL }\end{array}$ & 229 & Italy & $67-78$ & $\begin{array}{l}\mathrm{M}(n 83) \\
\mathrm{F}(n 146)\end{array}$ & \\
\hline $\begin{array}{l}\text { Wang et al. } \\
(2007)^{(58)}\end{array}$ & Correlation & $\begin{array}{l}\text { SBP, DBP, FPG, TC, } \\
\text { TAG, LDL, HDL }\end{array}$ & 1186 & Australia & Adult & $\mathrm{M}$ and $\mathrm{F}$ & $\begin{array}{l}\text { Aboriginal }(n 747) \text { or Torres } \\
\text { Strait Islanders }(n 439)\end{array}$ \\
\hline $\begin{array}{l}\text { Wu et al. } \\
\quad(2007)^{(59)}\end{array}$ & Correlation & $\begin{array}{l}\text { SBP, DBP, FPG, } \\
\text { HbA1c, TAG, HDL, }\end{array}$ & 411 & China & $\geq 40$ & $\begin{array}{l}\mathrm{M}(n 198) \\
\mathrm{F}(n 213)\end{array}$ & $\begin{array}{l}\text { Newly diagnosed T2D, } \\
\text { no history of diabetes } \\
\text { or diabetic complications }\end{array}$ \\
\hline $\begin{array}{l}\text { Yasmin \& } \\
\text { Mascie-Taylor } \\
(2000)^{(83)}\end{array}$ & Correlation & $\begin{array}{l}\text { SBP, DBP, TC, HDL, } \\
\text { LDL, TC:HDL, HDL:LDL }\end{array}$ & 368 & UK & $40-69$ & $\begin{array}{l}\mathrm{M}(n 165) \\
\mathrm{F}(n \text { 202) }\end{array}$ & \\
\hline $\begin{array}{l}\text { Gracey et al. } \\
(2007)^{(65)}\end{array}$ & $\begin{array}{l}\text { Compared } \\
\text { means above } \\
\text { and below } \\
\text { a cut-off }\end{array}$ & $\begin{array}{l}\text { SBP, DBP, FPI, FPG, } \\
\text { TC, TAG, LDL, HDL }\end{array}$ & 401 & $\begin{array}{l}\text { Australia, } \\
\text { Aborigine }\end{array}$ & Adult & $\begin{array}{l}\mathrm{M}(n 173) \\
\mathrm{F}(n 228)\end{array}$ & \\
\hline $\begin{array}{l}\text { Hsieh \& Yoshinaga } \\
(1995)^{(9)}\end{array}$ & $\begin{array}{l}\text { Linear } \\
\text { regression }\end{array}$ & $\begin{array}{l}\text { SBP, DBP, FPG, } \\
\text { HbA1c, TC, TAG, HDL }\end{array}$ & 1077 & Japan & $20-78$ & $\mathrm{~F}$ & \\
\hline $\begin{array}{l}\text { Hsieh \& Yoshinaga } \\
(1995)^{(10)}\end{array}$ & $\begin{array}{l}\text { Linear } \\
\text { regression }\end{array}$ & $\begin{array}{l}\text { SBP, DBP, } \\
\text { FPG, HbA1c, } \\
\text { TC, TAG, HDL, } \\
\text { risk factor index }\end{array}$ & 3131 & Japan & $22-82$ & M & \\
\hline $\begin{array}{l}\text { Lee et al. } \\
\qquad(2008)^{(72)}\end{array}$ & $\begin{array}{r}\text { Random } \\
\text { effects }\end{array}$ & $\begin{array}{l}\text { SBP, DBP, IFG, } \\
\text { HOMA-IR, } \\
\text { TAG, HDL }\end{array}$ & 1572 & Korea & $\geq 30$ & $\begin{array}{l}\mathrm{M}(n 577) \\
\quad \mathrm{F}(n 995)\end{array}$ & $\begin{array}{l}\text { Twin study } \\
\text { Same-sex twins }\end{array}$ \\
\hline $\begin{array}{l}\text { Khan et al. } \\
(2008)^{(70)}\end{array}$ & $\begin{array}{l}\text { Linear } \\
\quad \text { regression }\end{array}$ & SBP, DBP & 400 & Pakistan & Adult & $\begin{array}{l}\mathrm{M}(n 247) \\
\quad \mathrm{F}(n 153)\end{array}$ & Normotensive \\
\hline $\begin{array}{l}\text { Sakurai et al. } \\
(2006)^{(54)}\end{array}$ & $\begin{array}{l}\text { Linear } \\
\text { regression }\end{array}$ & Hypertension & 4557 & Japan & $35-59$ & $\begin{array}{l}\mathrm{M}(n \text { 2935); } \\
\mathrm{F}(n \text { 1622) }\end{array}$ & \\
\hline
\end{tabular}

SBP, systolic blood pressure; TC, total cholesterol; HOMA-IR, homeostasis model assessment of insulin resistance; M, male; F, female; KOPS, Kiel Obesity Prevention Study; FPG, fasting plasma glucose; DBP, diastolic blood pressure; FPI, fasting plasma insulin; 2hrG, $2 \mathrm{~h}$ blood glucose; MetS, metabolic syndrome; NHANES, National Health and Nutrition Examination Survey; HRT, hormone replacement therapy; HbA1c, glycated Hb; T2D, type 2 diabetes; MAP, mean arterial pressure; IFG, impaired fasting glycaemia. 


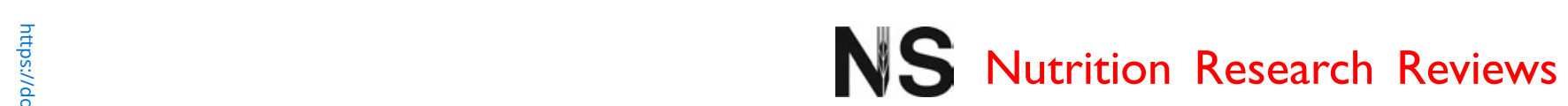

Table 6. Summary of the results of all cross-sectional studies in adults, analysing data with correlation or regression, by outcome

\begin{tabular}{|c|c|c|c|c|c|c|c|c|c|}
\hline \multirow[b]{2}{*}{ Outcome } & \multicolumn{3}{|c|}{ WHtR } & \multicolumn{3}{|c|}{ wc } & \multicolumn{3}{|c|}{ BMI } \\
\hline & $\begin{array}{l}\text { Summary } \\
\text { score }^{\star}\end{array}$ & $\begin{array}{l}\text { Significant } \\
\text { in all analyses }\end{array}$ & $\begin{array}{l}\text { Significant } \\
\text { in some or none } \\
\text { of the analyses }\end{array}$ & $\begin{array}{l}\text { Summary } \\
\text { score* }^{*}\end{array}$ & $\begin{array}{l}\text { Significant } \\
\text { in all analyses }\end{array}$ & $\begin{array}{l}\text { Significant } \\
\text { in some or none } \\
\text { of the analyses }\end{array}$ & $\begin{array}{l}\text { Summary } \\
\text { score }^{\star}\end{array}$ & $\begin{array}{l}\text { Significant } \\
\text { in all analyses }\end{array}$ & $\begin{array}{l}\text { Significant } \\
\text { in some or none } \\
\text { of the analyses }\end{array}$ \\
\hline FPI & $7 / 7$ & $\begin{array}{l}\text { In the population as } \\
\text { a wholele }(65,74,75,79) \\
\text { and divided by } \\
\text { sex }\end{array}$ & & $7 / 7$ & $\begin{array}{l}\text { In the population as } \\
\text { a wholel(65,74,75,79) } \\
\text { and divided by } \\
\text { sex }\end{array}$ & & $7 / 7$ & $\begin{array}{l}\text { In the population as } \\
\text { a wholele }(65,74,75,79) \\
\text { and divided } \\
\text { by sex }(66,72,80)\end{array}$ & \\
\hline FPG & $12 / 18$ & 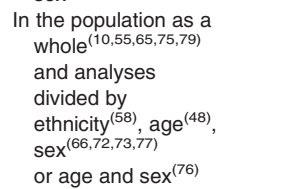 & $\begin{array}{l}\text { Not in whole study } \\
\text { population } \\
\text { in women, } \\
\text { but not men } \\
(61,82)\end{array}$ & $10 / 16$ & $\begin{array}{l}\text { In the population as } \\
\text { a whole } e^{(65,75,79)} \text { and } \\
\text { analyses divided by } \\
\text { ethnicitity(58) age }^{(48)}, \\
\text { sex }^{(66,72,73,77)} \text { or age } \\
\text { and sex }{ }^{(76)}\end{array}$ & $\begin{array}{l}\text { Not in whole study } \\
\text { population } \\
\text { in women, } \\
\text { but not men } \\
(61,82)\end{array}$ & $12 / 16$ & 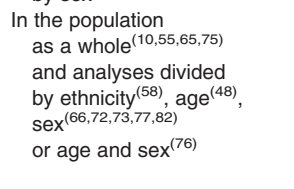 & $\begin{array}{l}\text { Not in whole study } \\
\text { population } 57,62,74) \\
\text { in women but } \\
\text { not } \text { men }^{(61)}\end{array}$ \\
\hline HOMA-IR & $4 / 4$ & $\begin{array}{l}\text { In the population as } \\
\text { a whole }(60,75) \text { and } \\
\text { divided by sex }{ }^{(61,72)}\end{array}$ & & $4 / 4$ & $\begin{array}{l}\text { In the population as a } \\
\text { whole }(60,75) \text { and } \\
\text { divided by sex }{ }^{(61,72)}\end{array}$ & & $4 / 4$ & $\begin{array}{l}\text { In the population as } \\
\text { a whole }(60,75) \text { and } \\
\text { divided by sex }{ }^{(61,72)}\end{array}$ & \\
\hline $2 \mathrm{hrG}$ & $4 / 6$ & $\begin{array}{l}\text { In analyses divided } \\
\text { by sex }{ }^{(66,73,76,78)}\end{array}$ & $\begin{array}{l}\text { Not in whole study } \\
\text { population }{ }^{(79)}, \\
\text { in women, } \\
\text { but not } \text { men }^{(82)}\end{array}$ & $3 / 6$ & $\begin{array}{l}\text { In analyses divided } \\
\text { by sex }{ }^{(66,73,78)}\end{array}$ & $\begin{array}{l}\text { Not in whole study } \\
\text { population (79), } \\
\text { in women, but not } \\
\text { men }{ }^{(82)} \text {, in women } \\
\text { and young men, } \\
\text { but not old men } \\
\text { (76) }\end{array}$ & $3 / 5$ & $\begin{array}{l}\text { In analyses divided } \\
\text { by sex } x^{(66,73,78)}\end{array}$ & $\begin{array}{l}\text { In women, but not men }{ }^{(82)} \text {, } \\
\text { in old women and } \\
\text { young men, but not } \\
\text { old men and young } \\
\text { women }{ }^{(76)}\end{array}$ \\
\hline HbA1c & $3 / 6$ & $\begin{array}{l}\text { In the population as } \\
\text { a whole }{ }^{(9,10)} \text { and } \\
\text { divided by sex }^{(59)}\end{array}$ & $\begin{array}{l}\text { Not in whole study } \\
\text { population }{ }^{(75)}, \\
\text { in women, } \\
\text { but not men }{ }^{(57,76)}\end{array}$ & $2 / 4$ & $\begin{array}{l}\text { In the population as a } \\
\text { whole }{ }^{(75)} \text { and divided } \\
\text { by } \operatorname{sex}^{(59)}\end{array}$ & $\begin{array}{l}\text { In women, but not } \\
\text { men }{ }^{(57)} \text {, in women } \\
\text { and young men, } \\
\text { but not old men } \\
\text { (76) }\end{array}$ & $3 / 6$ & $\begin{array}{l}\text { In the population as } \\
\text { a whole } e^{(9,10)} \text { and } \\
\text { divided by sex }^{(59)}\end{array}$ & $\begin{array}{l}\text { Not in whole study } \\
\text { population } \\
\text { in women, }{ }^{(57,5)}, \\
\text { not men }{ }^{(76)}\end{array}$ \\
\hline SBP & $21 / 22$ & $\begin{array}{l}\text { In the population as a } \\
\text { whole(9, } 10,55,57,60,63,65,75) \\
\text { and analyses divided by } \\
\text { ethnicityt(58), age } \\
\text { sex }^{(54,45,59,61,66,70,72,77,82,83)} \\
\text { or age and sex }{ }^{(47,76)}\end{array}$ & $\begin{array}{l}\text { In non-hypertensives } \\
\text { but not hypertensives }\end{array}$ & $16 / 19$ & 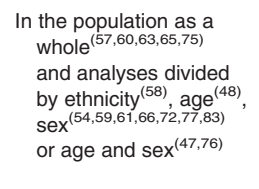 & $\begin{array}{l}\text { In non-hypertensives } \\
\text { but not } \\
\text { hypertensives } \\
\text { in men but not } \\
\text { women } \\
\text { in women but } \\
\text { not men } \\
\text { (82) }\end{array}$ & $19 / 22$ & 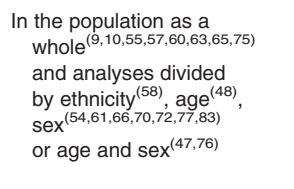 & $\begin{array}{l}\text { In non-hypertensives } \\
\text { but not } \\
\text { hypertensives } \\
\text { in men but not, } \\
\text { women }{ }^{(79)} \text {, in women } \\
\text { but not men }{ }^{(82)}\end{array}$ \\
\hline DBP & $18 / 21$ & 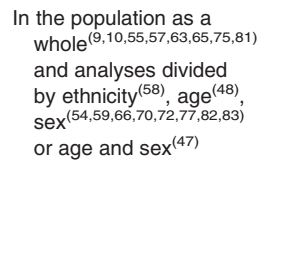 & $\begin{array}{l}\text { In non-hypertensives } \\
\text { but not in } \\
\text { hypertensives } \\
\text { significant in men } \\
\text { but not women } \\
\text { in young women, } \\
\text { but not men } \\
\text { or old women } \\
{ }^{(76)}\end{array}$ & $15 / 19$ & $\begin{array}{l}\text { In the population as a } \\
\text { whole }(57,63,65,75,81) \text { and } \\
\text { analyses divided by } \\
\text { ethnicity(y) }^{(58)} \text { age } \mathrm{e}^{(48)} \\
\text { sex }^{(54,59,66,70,77,83)} \\
\text { or age and sex }{ }^{(47)}\end{array}$ & $\begin{array}{l}\text { In non-hypertensives } \\
\text { but not } \\
\text { hypertensives } \\
\text { significant in } \\
\text { men but } \\
\text { women } \\
\text { wot } \\
\text { but not men } \text { men }^{(82)} \text {, } \\
\text { in young men and } \\
\text { women, but not in old } \\
\text { men and women } \\
\text { (76) }\end{array}$ & $19 / 21$ & $\begin{array}{l}\text { In the population as a } \\
\text { whole }{ }^{(9,10,55,57,63,65,75,81)} \\
\text { and analyses divided } \\
\text { by ethnicity } \\
\text { sex } x^{(54,59,69,66,70,72,7,7,82,88,83)} \\
\text { or age and sex }{ }^{(47)}\end{array}$ & $\begin{array}{l}\text { In non-hypertensives but } \\
\text { not hypertensives }{ }^{(71)}, \\
\text { in women, but not } \\
\text { in men }\end{array}$ \\
\hline TC & $8 / 16$ & $\begin{array}{l}\text { In the population as a } \\
\text { whole } e^{(9,10,55,60,62,65)} \\
\text { and analyses divided } \\
\text { by age } \mathrm{e}^{(48)} \text { or sex } \mathrm{x}^{(66)}\end{array}$ & $\begin{array}{l}\text { Not in whole study } \\
\text { population (57,58,74,75,82), } \\
\text { in men but not } \\
\text { women }{ }^{(61,80,83)}\end{array}$ & $5 / 13$ & $\begin{array}{l}\text { In the population as a } \\
\text { whole }(60,62,65) \text { and } \\
\text { analyses divided } \\
\text { by age } e^{(48)} \text { or } \operatorname{sex}^{(66)}\end{array}$ & 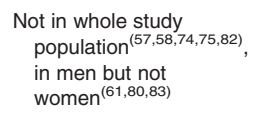 & $8 / 16$ & $\begin{array}{l}\text { In the population as a } \\
\text { whole } e^{(9,10,55,60,62,65)} \\
\text { and analyses divided } \\
\text { by age } \text { a }^{(48)} \text { or sex } \operatorname{sex}^{(66)}\end{array}$ & $\begin{array}{l}\text { Not in whole study popula- } \\
\text { tion } n^{(57,58,74,75,80,82,83)} \\
\text { in men but not women }\end{array}$ \\
\hline TAG & $19 / 21$ & $\begin{array}{l}\text { In the population as a } \\
\text { whole }{ }^{(9,10,55,60,62,65,75,78,79)} \\
\text { and analyses divided } \\
\text { by ethnicity } \\
\text { sex } x^{\left(59,61,66,72,77,80, e^{(48)}\right.} \text {, } \\
\text { age and sex }{ }^{(76)}\end{array}$ & $\begin{array}{l}\text { Not significant in whole } \\
\text { study population }{ }^{(74)} \\
\text { significant in women } \\
\text { but not men }{ }^{(57)}\end{array}$ & $16 / 18$ & $\begin{array}{l}\text { In the population as a } \\
\text { whole }(60,62,65,74,75,78,79) \\
\text { and analyses divided } \\
\text { by ethnicitity }(58), \text { age } \\
\text { sex }\left({ }^{(48), 61,66,72,77,70,82)}\right. \\
\text { or age and sex } \\
{ }^{(76)}\end{array}$ & $\begin{array}{l}\text { Not in whole study } \\
\text { population }{ }^{(79)}, \\
\text { in women but } \\
\text { not men }{ }^{(57)}\end{array}$ & $20 / 20$ & 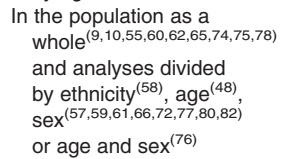 & \\
\hline LDL & $4 / 10$ & $\begin{array}{l}\text { In the population as a } \\
\text { whole } e^{(62,75)} \text { and } \\
\text { analyses } \\
\text { divided by age } \mathrm{e}^{(48)} \\
\text { or sex }{ }^{(66)}\end{array}$ & $\begin{array}{l}\text { Not in whole study } \\
\text { population (61,65,74,79), } \\
\text { in Torres Islanders } \\
\text { but not Aboriginals } \\
\text { in men but not } \\
\text { women } \\
\text { wor) }\end{array}$ & $5 / 10$ & $\begin{array}{l}\text { In the population as a } \\
\text { whole }(62,75,79) \text { and } \\
\text { analyses divided by } \\
\text { age } \mathrm{e}^{(48)} \text { or } \operatorname{sex}^{(66)}\end{array}$ & $\begin{array}{l}\text { Not in whole study } \\
\text { population } \\
\text { in Torres Islander, } \\
\text { but not Aboriginals } \\
\text { in men but not women }{ }^{(58)}\end{array}$ & $5 / 9$ & $\begin{array}{l}\text { In the population as a } \\
\text { whole }(62,65,75) \text { and } \\
\text { analyses divided by } \\
\text { age }^{(48)} \text { or } \operatorname{sex}^{(66)}\end{array}$ & $\begin{array}{l}\text { Not in whole study } \\
\text { population } 58,61,74) \\
\text { in men but not } \\
\text { women } \\
\text { wor) }\end{array}$ \\
\hline
\end{tabular}




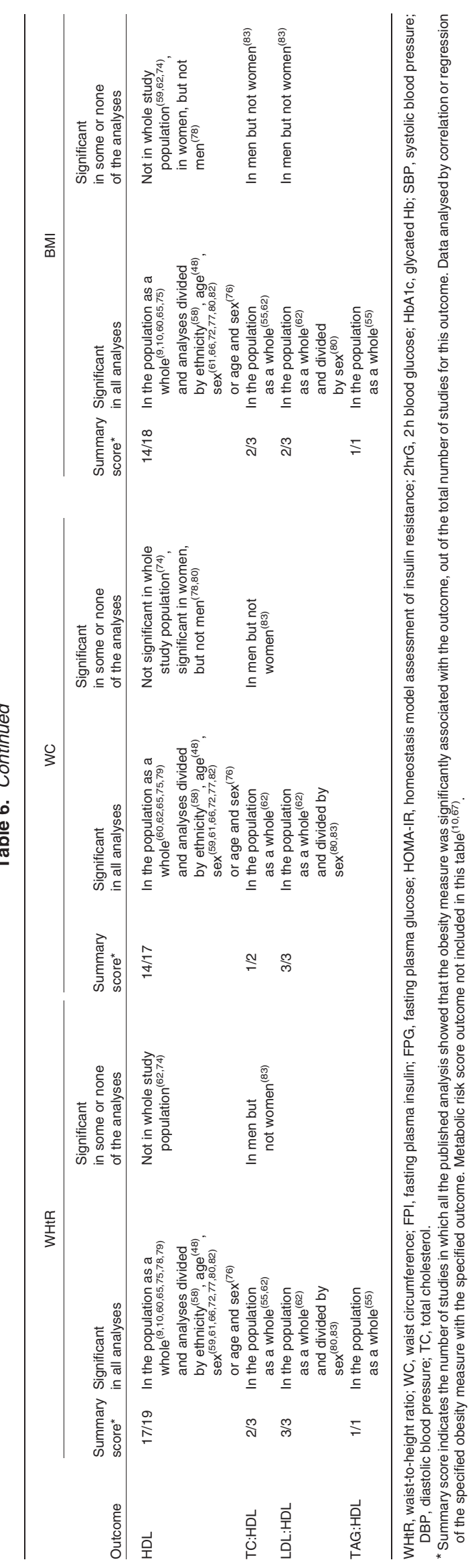

and WHtR serve as independent risk factors, some crosssectional studies have determined OR or relationships after adjustments for BMI. Harris et al. showed that odds of hypertension increased after adjustment for BMI through quintiles of WHtR and WC in White and African-American women, and in some groups of men ${ }^{(50)}$. OR were similar for WHtR and WC. Two other studies determined odds of various risk factors in groups with low or high BMI and/or low or high WHtR and showed that the group with high BMI and high WHtR were at the highest risk ${ }^{(51,52)}$.

\section{Prospective and cross-sectional studies in children}

A total of thirteen cross-sectional studies were conducted in children ${ }^{(84-96)}$, with one study also including a prospective analysis $^{(87)}$ (Table 7). Seven studies reported correlations, two linear regression and four reported OR above a particular boundary value. In general, studies showed good agreement in the magnitude and outcome of their analysis between WHtR, WC and BMI and outcomes of metabolic risk (Table 8).

For SBP, WHtR, WC and BMI were significantly associated in six of eight, five of six and five of seven studies, respectively. For DBP, WHtR, WC and BMI were significantly associated in three of six, three of four and three of five studies, respectively. Where relationships for SBP and DBP were determined in the same studies, relationships tended to be significant and stronger for SBP than DBP ${ }^{(85,87,89)}$.

For fasting plasma insulin, WHtR and BMI were significantly associated, in four of four studies, for all children, with WC significant in two of two studies. In one study, homeostasis model assessment of insulin resistance (HOMA-IR) was significantly correlated with WHtR, $\mathrm{WC}$ and BMI. The relationship between fasting plasma glucose and WHtR, WC and BMI was less convincing in the few studies where it was reported.

For TAG, WHtR was significantly associated in seven of seven studies, WC in three of four studies, and BMI in five of six studies. For HDL, WHtR was negatively associated in three of five studies, WC in three of three studies, and BMI in three of four studies. Of the lipid outcomes, similar to the results in adults, relationships with the anthropometric indices tended to be stronger for TAG and HDL. Hara et al. ${ }^{(85)}$ and Kahn et al. ${ }^{(95)}$ showed the only significant relationships for TC and WHtR, with three other studies showing non-significant relationships. For LDL, WHtR was significantly associated in six of seven studies, WC in two of four studies and BMI in two of six studies.

In addition to the results in Table 7, four studies determined the relationship between WHtR, WC and BMI and a 'metabolic factor score'. This was significant for WHtR in four of four studies, WC in three of three studies and for BMI in two of three studies.

\section{Summary of results for anthropometric indices as significant predictors of disease risk}

The data presented in the present systematic review, from both cross-sectional and prospective datasets, show that WHtR, WC and BMI are useful predictors of CVD and diabetes risk. The balance of evidence suggests that WHtR 


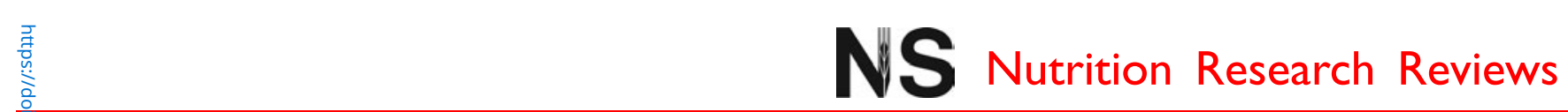

Table 7. Details of cross-sectional studies in children

\begin{tabular}{|c|c|c|c|c|c|c|c|}
\hline \multirow[b]{2}{*}{ Study reference } & \multirow[b]{2}{*}{ Study design: analysis type } & \multirow[b]{2}{*}{ Outcomes } & \multicolumn{5}{|c|}{ Population } \\
\hline & & & Subjects $(n)$ & Country & Age (years) & Sex & Inclusion criteria \\
\hline Botton et al. $(2007)^{(84)}$ & Correlation & $\begin{array}{l}\text { SBP, DBP, FPI, FPG, } \\
\text { TC, TAG, LDL, HDL }\end{array}$ & 452 & France & $8-17$ & $\mathrm{M}(n 235) ; \mathrm{F}(n 217)$ & \\
\hline Hara et al. $(2002)^{(85)}$ & Correlation & $\begin{array}{l}\text { SBP, DBP, TC, TAG, } \\
\text { LDL, HDL, atherogenic } \\
\text { index, CVD risk score }\end{array}$ & 888 & Japan & $9-13$ & $\mathrm{M}(n 447) ; \mathrm{F}(n 433)$ & \\
\hline Manios et al. (2008) ${ }^{(86)}$ & Correlation & FPI, HOMA-IR & 248 & Greece & $10-12$ & $\mathrm{M}$ and $\mathrm{F}$ & \\
\hline Mirzaei et al. $(2007)^{(87)}$ & $\begin{array}{l}\text { Prospective } \\
\text { ( } 3 \text { years) and } \\
\text { correlation }\end{array}$ & SBP, DBP & 1230,628 & Australia & $8-9$ & $\mathrm{M}(n 314) ; \mathrm{F}(n 314)$ & \\
\hline Ruiz et al. $(2007)^{(88)}$ & Correlation & SBP, DBP, MAP & 873 & $\begin{array}{l}\text { Estonia and } \\
\text { Sweden }\end{array}$ & $9-10$ & $\mathrm{M}(n$ 429); F (n 444) & \\
\hline Sung et al. (2007) ${ }^{(89)}$ & Correlation & $\begin{array}{l}\text { SBP, DBP, FPI, FPG, } \\
\text { TAG, HDL, LDL }\end{array}$ & 1055 & Hong Kong & $6-12$ & $\mathrm{M}(n 566) ; \mathrm{F}(n 489)$ & $\begin{array}{l}\text { Randomly } \\
\text { selected } \\
\text { plus some } \\
\text { overweight } \\
\text { children }\end{array}$ \\
\hline Teixeira et al. $(2001)^{(90)}$ & Correlation & $\begin{array}{l}\text { TC, TAG, HDL, LDL, } \\
\text { TC:HDL }\end{array}$ & 159 & Portugal & $10-15$ & $\mathrm{M}(n 72) ; \mathrm{F}(n 87)$ & \\
\hline Kahn et al. $(2005)^{(95)}$ & Linear regression & $\begin{array}{l}\text { Heart rate, SBP, } \\
\text { FPG, TC, TAG, } \\
\text { LDL, TC:HDL }\end{array}$ & 6652,821 & USA & $4-17$ & $\mathrm{M}$ and $\mathrm{F}$ & $\begin{array}{l}\text { NHANES III } \\
\text { Without diabetes }\end{array}$ \\
\hline Mesa et al. $(2006)^{(96)}$ & Linear regression & Lipid risk factor score & 524 & Spain & $15 \cdot 3 \pm 1 \cdot 4$ years & $\mathrm{F}(n$ 259); M (n 265) & \\
\hline Freedman et al. (2009) & OR, tertiles & $\begin{array}{l}\text { Metabolic score, TAG, } \\
\text { LDL, HDL, FPI, } \\
\text { SBP, DBP }\end{array}$ & 2501 & USA & $5-17$ & $\mathrm{M}(n 1200) ; \mathrm{F}(n$ 1301) & $\begin{array}{l}\text { Bogalusa } \\
\text { Heart Study }\end{array}$ \\
\hline Garnett et al. (2008) ${ }^{(92)}$ & OR, cut-offs & CVD risk cluster & 164 & Australia & Mean 14.9 (SD 0.2) & $\mathrm{M}(n 86) ; \mathrm{F}(n 78)$ & \\
\hline Maffeis et al. $(2008)^{(93)}$ & $\begin{array}{l}\text { OR, obesity } \\
\pm \text { WHtR/WC } \\
\text { category }\end{array}$ & Metabolic syndrome & 1479 & Italy & $5-15$ & $\mathrm{M}(n 740) ; \mathrm{F}(n 739)$ & \\
\hline Savva et al. $(2000)^{(94)}$ & OR, $>75$ th percentile & $\begin{array}{l}\text { High SBP, high TC, } \\
\text { high LDL, or } \\
\text { high TAG }\end{array}$ & 1987 & Cyprus & $10-14$ & $\mathrm{M}(n$ 1037); F ( $n 950)$ & \\
\hline
\end{tabular}

SBP, systolic blood pressure; DBP, diastolic blood pressure; FPI, fasting plasma insulin; FPG, fasting plasma glucose; TC, total cholesterol; M, male; F, female; HOMA-IR, homeostasis model assessment of insulin resistance; MAP, mean arterial pressure; WHtR, waist-to-height ratio; WC, waist circumference; NHANES III, Third National Health and Nutrition Examination Survey. 


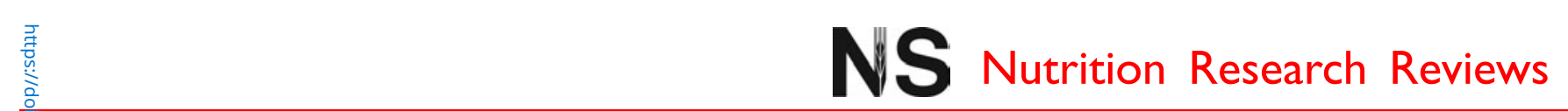

Table 8. Summary of the results of all cross-sectional studies in children (OR and correlation), by outcome

\begin{tabular}{|c|c|c|c|c|c|c|c|c|c|}
\hline \multirow[b]{2}{*}{ Outcome } & \multicolumn{3}{|c|}{ WHtR } & \multicolumn{3}{|c|}{ WC } & \multicolumn{3}{|c|}{ BMI } \\
\hline & $\begin{array}{l}\text { Summary } \\
\text { score* }^{*}\end{array}$ & $\begin{array}{l}\text { Significant } \\
\text { in all analyses }\end{array}$ & $\begin{array}{l}\text { Significant in some } \\
\text { or none of the } \\
\text { analyses }\end{array}$ & $\begin{array}{l}\text { Summary } \\
\text { score* }^{*}\end{array}$ & $\begin{array}{l}\text { Significant } \\
\text { in all analyses }\end{array}$ & $\begin{array}{l}\text { Significant in } \\
\text { some or none } \\
\text { of the analyses }\end{array}$ & $\begin{array}{l}\text { Summary } \\
\text { score }^{*}\end{array}$ & $\begin{array}{l}\text { Significant in } \\
\text { all analyses }\end{array}$ & $\begin{array}{l}\text { Significant in } \\
\text { some or none } \\
\text { of the analyses }\end{array}$ \\
\hline FPI & $4 / 4$ & $\begin{array}{l}\text { In girls and } \\
\text { boys }(84,86,89,91)\end{array}$ & & $2 / 2$ & $\begin{array}{l}\text { In girls and } \\
\text { boys }^{(86,89)}\end{array}$ & & $4 / 4$ & $\begin{array}{l}\text { In girls and } \\
\text { boys }(84,86,89,91)\end{array}$ & \\
\hline FPG & $2 / 3$ & $\begin{array}{l}\text { In girls and } \\
\text { boys }^{(89,95)}\end{array}$ & $\begin{array}{l}\text { In girls but } \\
\text { not boys }\end{array}$ & $0 / 1$ & & $\begin{array}{l}\text { In girls but } \\
\text { not boys }\end{array}$ & $1 / 3$ & $\begin{array}{l}\text { In girls and } \\
\text { boys }^{(95)}\end{array}$ & $\begin{array}{l}\text { In girls but not } \\
\text { boys }^{(84,89)}\end{array}$ \\
\hline HOMA-IR & $1 / 1$ & In girls and boys ${ }^{(86)}$ & & $1 / 1$ & $\begin{array}{l}\text { In girls and } \\
\text { boys }^{(86)}\end{array}$ & & $1 / 1$ & $\begin{array}{l}\text { In girls and } \\
\text { boys } \text { s }^{(86)}\end{array}$ & \\
\hline SBP & $6 / 8$ & $\begin{array}{l}\text { In girls and } \\
\text { boys } \\
(85,87,89,91,94,95)\end{array}$ & $\begin{array}{l}\text { In girls but } \\
\text { not boys } \\
\text { in boys but not } \\
\text { girls }^{(84)}\end{array}$ & $5 / 6$ & $\begin{array}{l}\text { In girls and } \\
\text { boys }^{(85,87,89,94)}\end{array}$ & $\begin{array}{l}\text { In girls but } \\
\text { not boys }\end{array}$ & $5 / 7$ & $\begin{array}{l}\text { In girls and } \\
\text { boys }^{(87-89,94,95)}\end{array}$ & $\begin{array}{l}\text { In boys but not } \\
\text { girls }^{(84)} \text {, not in } \\
\text { girls or boys } \\
{ }^{(91)}\end{array}$ \\
\hline DBP & $3 / 6$ & $\begin{array}{l}\text { In girls and } \\
\text { boys }^{(84,85,89)}\end{array}$ & $\begin{array}{l}\text { In boys but not } \\
\text { girls }{ }^{(87)} \text {, in girls } \\
\text { but not boys } \\
\text { not in girls } \\
\text { or boys } \\
\left({ }^{(81)}\right.\end{array}$ & $3 / 4$ & $\begin{array}{l}\text { In girls and } \\
\text { boys }^{(85,87,89)}\end{array}$ & $\begin{array}{l}\text { In girls but } \\
\text { not boys }{ }^{(88)}\end{array}$ & $3 / 5$ & $\begin{array}{l}\text { In girls and } \\
\text { boys }^{(84,87,89)}\end{array}$ & $\begin{array}{l}\text { In girls but not } \\
\text { boys }{ }^{(88)}, \text { not in } \\
\text { girls or boys }{ }^{(91)}\end{array}$ \\
\hline TC & $2 / 5$ & $\begin{array}{l}\text { In girls and } \\
\text { boys }{ }^{(85,95)}\end{array}$ & $\begin{array}{l}\text { In girls but not } \\
\text { boys } \\
\text { but not girls boys } \\
\text { not in girls } \\
\text { or boys } \\
\left({ }^{(90)}\right.\end{array}$ & $0 / 3$ & & $\begin{array}{l}\text { Not in girls or } \\
\text { boys } \\
\text { in boys but } \\
\text { not girls }{ }^{(94)}\end{array}$ & $1 / 4$ & $\begin{array}{l}\text { In girls and } \\
\text { boys } \text { s }^{(95)}\end{array}$ & $\begin{array}{l}\text { Not in girls } \\
\text { or boys } \\
\text { in boys but } \\
\text { not girls }^{(94)}\end{array}$ \\
\hline TAG & $7 / 7$ & $\begin{array}{l}\text { In girls and } \\
\text { boys } \\
(84,85,89-91,94,95)\end{array}$ & & $3 / 4$ & $\begin{array}{l}\text { In girls and } \\
\text { boys }(85,89,94)\end{array}$ & $\begin{array}{l}\text { Not in girls } \\
\text { or boys }\end{array}$ & $5 / 6$ & $\begin{array}{l}\text { In girls and } \\
\text { boys }{ }^{(84,85,89,94,95)}\end{array}$ & $\begin{array}{l}\text { Not in girls } \\
\text { or boys }^{(91)}\end{array}$ \\
\hline LDL & $6 / 7$ & $\begin{array}{l}\text { In girls and } \\
\text { boys }{ }^{(84,85,89,91,94,95)}\end{array}$ & $\begin{array}{l}\text { Not in girls } \\
\text { or boys }\end{array}$ & $2 / 4$ & $\begin{array}{l}\text { In girls and } \\
\text { boys }^{(85,94)}\end{array}$ & $\begin{array}{l}\text { In boys but } \\
\text { not girls } \\
\text { not in girls } \\
\text { or boys }^{(90)}\end{array}$ & $2 / 6$ & $\begin{array}{l}\text { In girls and } \\
\text { boys }^{(85,94)}\end{array}$ & $\begin{array}{l}\text { In girls but not } \\
\text { boys } \\
\text { but not girls } \\
\text { not in girls } \\
\text { or boys } \\
\text { or }{ }^{(81,95)}\end{array}$ \\
\hline HDL & $3 / 5$ & $\begin{array}{l}\text { In girls and } \\
\text { boys }^{(84,85,89)}\end{array}$ & $\begin{array}{l}\text { Not in girls } \\
\text { or boys }^{(90,91)}\end{array}$ & $3 / 3$ & $\begin{array}{l}\text { In girls and } \\
\text { boys }^{(85,89,90)}\end{array}$ & & $3 / 4$ & $\begin{array}{l}\text { In girls and } \\
\text { boys }^{(84,85,89)}\end{array}$ & $\begin{array}{l}\text { Not in girls } \\
\text { or boys }^{(91)}\end{array}$ \\
\hline TC:HDL & $1 / 2$ & In girls and boys ${ }^{(95)}$ & $\begin{array}{l}\text { Not in girls } \\
\text { or boys }^{(90)}\end{array}$ & $0 / 1$ & & $\begin{array}{l}\text { Not in girls } \\
\text { or boys }\end{array}$ & $2 / 2$ & $\begin{array}{l}\text { In girls and } \\
\text { boys }^{(90,95)}\end{array}$ & \\
\hline
\end{tabular}

WHtR, waist-to-height ratio; WC, waist circumference; FPI, fasting plasma insulin; FPG, fasting plasma glucose; HOMA-IR, homeostasis model assessment of insulin resistance; SBP, systolic blood pressure; DBP, diastolic blood pressure; TC, total cholesterol. ${ }^{\star}$ Summary score indicates the number of studies in which all the published analysis showed that the obesity measure was significantly associated with the outcome, out of diastolic blood pressure; TC, total cholesterol. ${ }^{\star}$ Summary score indicates the number of studies in which all the published analysis showed that the obesity measure was significantly associated with the outcome, out of
the total number of studies for this outcome. Results not tabulated are those where the outcome was not a commonly reported one: lipid factor risk score ${ }^{(96)}$, risk factor cluster ${ }^{(92)}$, metabolic syndrome ${ }^{(93)}$, total risk factors $^{(91)}$, atherogenic index and CVD risk score ${ }^{(85)}$. 
and $\mathrm{WC}$ are stronger predictors than BMI. In studies where adjustment has been made, WHtR and WC were also predictors of outcomes independent of BMI.

Collating results for all prospective studies (Table 9) showed that WHtR and WC were a significant risk factor in $72 \%$ of all outcomes, and BMI in $58 \%$ of all outcomes. The collations of data from the cross-sectional studies in adults indicate that the WHtR is a significant predictor in $77 \%$ of all outcomes, WC is a significant predictor in $72 \%$ of all outcomes and BMI in $77 \%$ of all outcomes. Similar collations of data from the cross-sectional studies in children indicate the $\mathrm{WHtR}$ is a significant predictor in $73 \%$ of all outcomes, WC is a significant predictor in $66 \%$ of all outcomes and BMI in $64 \%$ of all outcomes. Combining all cross-sectional studies in adults and children (Table 9) indicates that the WHtR is a significant predictor in $76 \%$ of all outcomes, WC is a significant predictor in $71 \%$ of all outcomes and BMI in $74 \%$ of all outcomes.

Limitations of prospective and cross-sectional analyses of anthropometric indices. From the evidence presented so far, WHtR and WC appear to be as good, if not better than BMI in predicting metabolic risk. However, while some prospective studies show higher OR or RR with one or other measure, it is difficult to determine whether there is an advantage for clinical practice from this type of analysis of data since most statistical analysis is derived from either continuous relationships or boundary values based on the data itself (per $1 \mathrm{SD}$, by tertiles, quartiles, quintiles), rather than practical boundary values for diagnostic or screening purposes, for example, BMI $\geq 30 \mathrm{~kg} / \mathrm{m}^{2}$, WC $\geq 88$ or $102 \mathrm{~cm}$ and WHtR $\geq 0 \cdot 5$. Further analysis is therefore required to determine whether either $\mathrm{WC}$ or $\mathrm{WHtR}$ has an advantage over the other for practical purposes.

\section{Practical use of anthropometric indices for screening purposes}

Information from receiver operating characteristic analysis. ROC data provide important information, assessing and comparing the diagnostic accuracy of different tests, for a particular outcome. In conducting the above systematic review, a number of studies undertaking ROC analyses and reporting area under ROC (AUROC) curve data were found. This information was used to further compare the usefulness of WHtR and WC as practical diagnostic tools.

At the stage of searching full papers, thirty-one papers with AUROC analysis were identified, twenty of which $^{(1,23,24,26,28,32,34,36,48,51,54,56,60,61,63,65,66,72,73,77)}$ also met criteria for the review and eleven ${ }^{(97-107)}$ of which were excluded from the systematic review at that stage (as they did not report the required analysis type and usually instead focused on AUROC analysis). Of these thirty-one papers with AUROC analysis, four did not report AUROC values $^{(48,63,98,107)}$, but only reported the optimum cut-off values and one did not report values for $\mathrm{WC}^{(100)}$, leaving twenty-six studies reporting full ROC information for different ethnic groups and age ranges. Details of the eighteen studies that met the criteria for the full review are included in the appropriate Tables 1, 3 or 7. Details of the other eight studies are included in Table 10.

Comparison of receiver operating characteristic values: support for waist-to-height ratio as a screening tool. A direct comparison of the AUROC values for WHtR and WC in the twenty-six papers included a total of 147 separate analyses, seventy-one in men, seventy-two in women, and four not divided by sex for a variety of outcomes related to diabetes and CVD risk. These four studies were included in

Table 9. Broad summary of the results of all prospective studies in adults and cross-sectional studies in adults and children by outcome (by number of significant/total number of studies, and percentage)

\begin{tabular}{|c|c|c|c|c|}
\hline Study design & Outcome & $\mathrm{WHtR}$ & WC & BMI \\
\hline \multirow[t]{7}{*}{ Prospective: adults } & Diabetes & $\begin{array}{l}6 / 9 \\
67 \%\end{array}$ & $\begin{array}{l}6 / 9 \\
67 \%\end{array}$ & $\begin{array}{l}5 / 9 \\
56 \%\end{array}$ \\
\hline & CVD events & $7 / 9$ & $7 / 9$ & $6 / 8$ \\
\hline & (fatal and non-fatal) & $78 \%$ & $78 \%$ & $75 \%$ \\
\hline & All stroke & $2 / 2$ & $2 / 2$ & $1 / 2$ \\
\hline & & $100 \%$ & $100 \%$ & $50 \%$ \\
\hline & Hypertension, & $3 / 5$ & $3 / 5$ & $2 / 5$ \\
\hline & SBP and DBP & $60 \%$ & $60 \%$ & $40 \%$ \\
\hline \multirow[t]{2}{*}{ All prospective studies } & Total & $18 / 25$ & $18 / 25$ & $14 / 24$ \\
\hline & & $72 \%$ & $72 \%$ & $58 \%$ \\
\hline \multirow[t]{8}{*}{ Cross-sectional: adults } & Diabetes & $36 / 48$ & $32 / 43$ & $35 / 45$ \\
\hline & & $75 \%$ & $74 \%$ & $78 \%$ \\
\hline & Lipid & $64 / 89$ & $51 / 79$ & $60 / 86$ \\
\hline & & $72 \%$ & $65 \%$ & $70 \%$ \\
\hline & CVD, hypertension, & $50 / 57$ & $41 / 51$ & $49 / 57$ \\
\hline & SBP, DBP & $88 \%$ & $80 \%$ & $86 \%$ \\
\hline & Total & $150 / 194$ & $124 / 173$ & $144 / 188$ \\
\hline & & $77 \%$ & $72 \%$ & $77 \%$ \\
\hline Cross-sectional: children & All health outcomes & $35 / 48$ & $19 / 29$ & $27 / 42$ \\
\hline & & $73 \%$ & $66 \%$ & $64 \%$ \\
\hline \multirow[t]{2}{*}{ All cross-sectional studies } & Total & $185 / 242$ & $143 / 202$ & $171 / 230$ \\
\hline & & $76 \%$ & $71 \%$ & $74 \%$ \\
\hline
\end{tabular}

WHtR, waist-to-height ratio; WC, waist circumference; SBP, systolic blood pressure; DBP, diastolic blood pressure. 


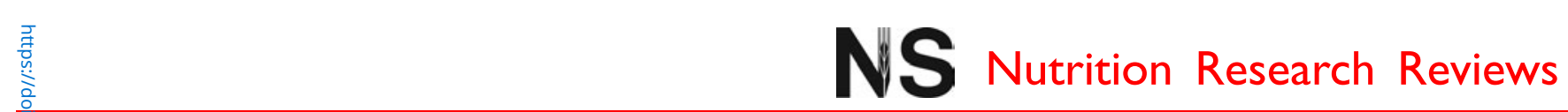

Table 10. Details of studies contributing data to the receiver operator characteristic (ROC) analysis (not previously tabulated)

\begin{tabular}{|c|c|c|c|c|c|c|c|}
\hline \multirow[b]{2}{*}{ Study reference } & \multicolumn{2}{|c|}{ Study design } & \multicolumn{5}{|c|}{ Population } \\
\hline & $\begin{array}{l}\text { Anthropometric } \\
\text { indices analysed } \\
\text { in ROC }\end{array}$ & Outcomes & Subjects $(n)$ & Country & Age (years) & Sex & Comments \\
\hline $\begin{array}{l}\text { Aekplakorn } \\
\text { et al. }(2006)^{(97)}\end{array}$ & WHtR, WC and BMI & $\begin{array}{l}\text { SBP, DBP, FPG, TC, } \\
\text { TAG, HDL, LDL, } \\
\text { TC:HDL, MetS }\end{array}$ & 5305 & Thailand & $>35$ & $\begin{array}{l}\mathrm{M}(n \text { 2093); } \\
\mathrm{F}(n 3212)\end{array}$ & $\begin{array}{l}\text { Inter Asia national } \\
\text { cross-sectional survey }\end{array}$ \\
\hline $\begin{array}{l}\text { Berber } \\
\text { et al. }(2001)^{(98)}\end{array}$ & WHtR, WC and BMI & $\begin{array}{l}\text { Diabetes, hypertension, } \\
\text { DYSLIP }\end{array}$ & 8365 & Mexico & $>20$ & $\begin{array}{l}\mathrm{M}(n \text { 2426); } \\
\mathrm{F}(n 5939)\end{array}$ & $\begin{array}{l}\text { Cross-sectional study } \\
\text { of general hospital } \\
\text { workers (1994 to 2000) }\end{array}$ \\
\hline Diaz et al. $(2007)^{(99)}$ & WHtR, WC and BMI & Diabetes & 11624 & USA, England & $>40$ for ROC & $\begin{array}{l}\mathrm{M}(n 5561) \\
\mathrm{F}(n 6063)\end{array}$ & $\begin{array}{l}\text { National cross-sectional } \\
\text { surveys in USA } \\
\text { (NHANES) and } \\
\text { England (HSE) } \\
(2003-4)\end{array}$ \\
\hline Ko et al. $(1999)^{(102)}$ & WHtR, WC and BMI & $\begin{array}{l}\text { Diabetes, hypertension, } \\
\text { DYSLIP }\end{array}$ & 1513 & Hong Kong & $37.5 \pm 9.2$ & $\begin{array}{l}\mathrm{M}(n 910) \\
\mathrm{F}(n 603)\end{array}$ & $\begin{array}{l}\text { Cross-sectional study } \\
\text { in Hong Kong Chinese } \\
\text { working population, } \\
1991\end{array}$ \\
\hline Lin et al. $(2002)^{(103)}$ & WHtR, WC and BMI & $\begin{array}{l}\text { SBP, DBP, FPG, TC, } \\
\text { TAG, HDL, LDL, } \\
\text { TC:HDL, MetS }\end{array}$ & 55563 & Taiwan & $\begin{array}{r}\text { M } 37.3 \pm 10 \cdot 9 \\
\quad \text { F } 37.0 \pm 11.1\end{array}$ & $\begin{array}{l}\mathrm{M}(n 26359) \\
\mathrm{F}(n 29204)\end{array}$ & $\begin{array}{l}\text { Cross-sectional study } \\
\text { in health screening } \\
\text { centres } 1998 \text { to } 2000\end{array}$ \\
\hline $\begin{array}{l}\text { Mansour \& Al-Jazairi } \\
(2007)^{(105)}\end{array}$ & WHtR, WC and BMI & Diabetes, hypertension & 12986 & Iraq & $45 \cdot 6 \pm 15 \cdot 7$ & $\begin{array}{l}\mathrm{M}(n \text { 6693); } \\
\mathrm{F}(n 6293)\end{array}$ & $\begin{array}{l}\text { Community-based } \\
\text { cross-sectional study }\end{array}$ \\
\hline $\begin{array}{l}\text { Mirmiran et al. } \\
\quad(2004)^{(106)}\end{array}$ & WHtR, WC and BMI & $\begin{array}{l}\text { Diabetes, hypertension, } \\
\text { DYSLIP, MetS }\end{array}$ & 10522 & Iran & $18-74$ & $\begin{array}{l}\mathrm{M}(n \text { 4449); } \\
\mathrm{F}(n \text { 6073) }\end{array}$ & $\begin{array}{l}\text { Cross-sectional study } \\
\text { within framework of } \\
\text { Tehran Lipid and } \\
\text { Glucose Study }\end{array}$ \\
\hline $\begin{array}{l}\text { Shimajiri } \\
\text { et al. }(2008)^{(107)}\end{array}$ & WHtR, WC & MetS & 5571 & Japan & $\begin{array}{r}\text { M } 47 \pm 12 \\
\text { F } 44 \pm 13\end{array}$ & $\begin{array}{l}\mathrm{M}(n \text { 3148); } \\
\mathrm{F}(n 2423)\end{array}$ & $\begin{array}{l}\text { Cross-sectional study } \\
\text { at health centre } \\
\text { check-ups }\end{array}$ \\
\hline
\end{tabular}



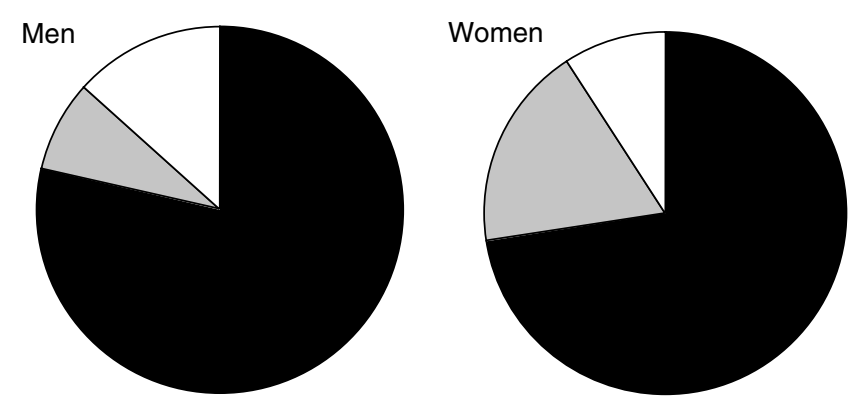

Fig. 2. Ranking of area under receiver operator characteristic (AUROC) values in twenty-six study populations, for 147 separate outcome analyses (seventy-one men, seventy-two women, four included in both). (ロ), Waist-to-height ratio (WHtR) AUROC > waist circumference (WC) AUROC; ( $\square$ ), WHIR AUROC = WC AUROC; $(\square)$, WC AUROC $>$ WHtR AUROC.

the data for both men and women, giving a total of seventyfive and seventy-six analyses in men and women, respectively. In order to describe the AUROC analyses, we grouped the studies according to whether AUROC values were higher for WHtR, higher for WC or equal for the two outcomes. Figure 2 shows that WHtR, compared with WC, had a higher AUROC value in the vast majority of studies, higher in fifty-eight of seventy-four analyses in men $(78 \%)$ and for fifty-four of seventy-five analyses in women $(72 \%)$.
In several cases, AUROC was the same for WHtR and WC and so WHtR was higher than or equal to WC in sixty-four of seventy-four analyses in men $(86 \%)$ and for sixty-eight of seventy-five analyses in women (91\%).

Mean AUROC calculations were weighted for study size. Across the 147 individual analyses in the thirty-one papers, in men and women, the mean AUROC values were 0.704, 0.693 and 0.670 for WHtR, WC and BMI, respectively. Analyses were then grouped by outcome: diabetes, CVD, insulin resistance, hypertension, dyslipidaemia, and the metabolic syndrome. Figure 3 shows the mean AUROC values within each of these categories for men (Fig. 3(a)) and women (Fig. 3(b)). For all outcome categories, in men and women, WHtR showed the highest AUROC value followed by WC, then BMI, indicating that WHtR provides a good screening tool overall for metabolic risk and also for diabetes, CVD and their respective risk factors.

\section{Use of receiver operator characteristic data to suggest boundary values for anthropometric indices}

ROC curves can also be used to derive and suggest 'boundary' or 'cut-off' values for anthropometric indices when they are used for practical screening purposes. We prefer the term boundary values, because they are used within a continuous relationship, and hence use it here.
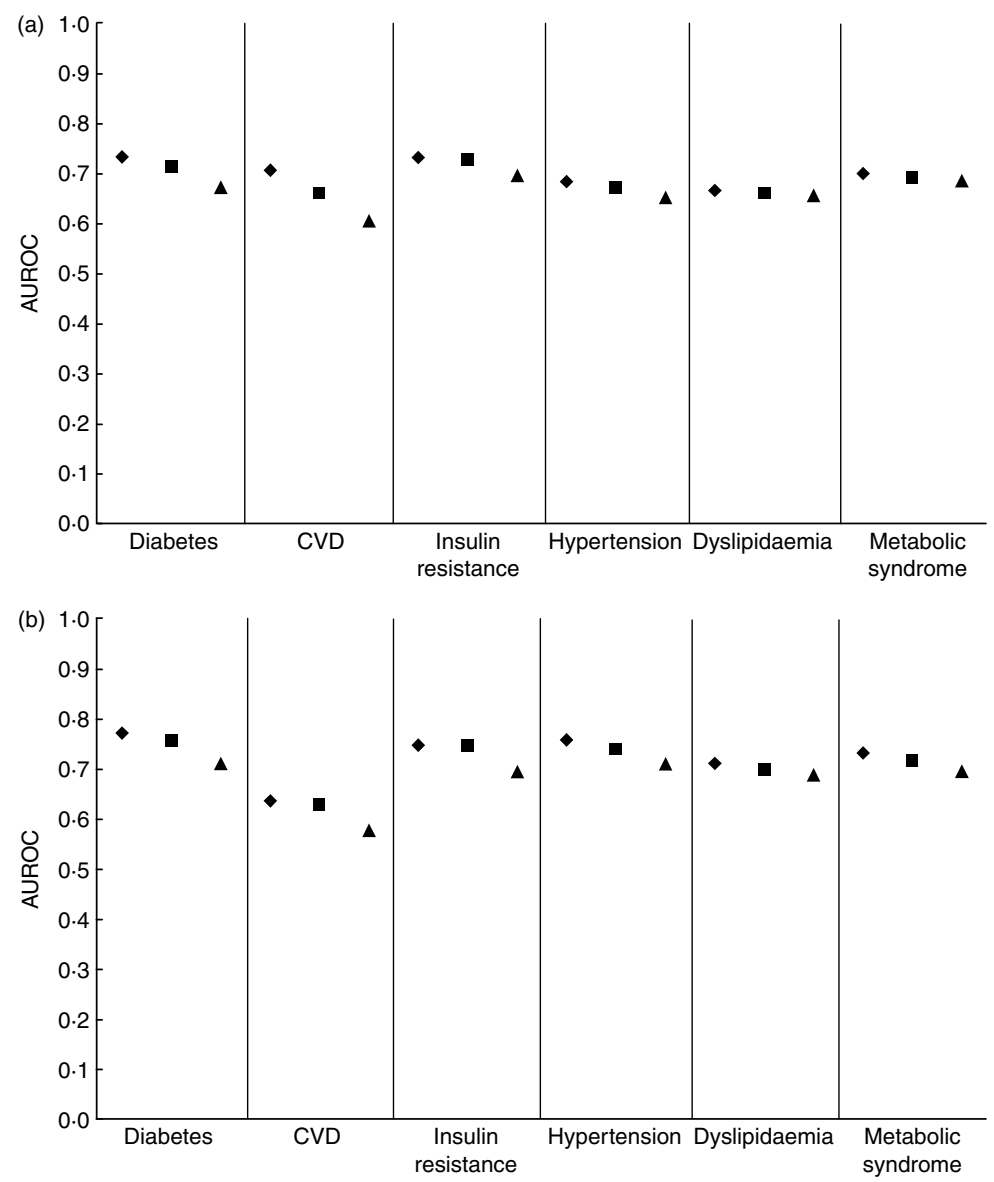

Fig. 3. Mean (weighted for sample size) area under receiver operator characteristic (AUROC) values for waist-to-height ratio ( $\bullet$ ), waist circumference $(\boldsymbol{\square})$ and BMI $(\boldsymbol{\Lambda})$, divided by outcome in men (a) and women (b). 
In a ROC curve, the sensitivity (relating to the ability of the anthropometric indices to predict health outcomes in this case) is plotted against the reciprocal of specificity. The AUROC is then calculated and the higher mean value for AUROC indicates better discrimination by the anthropometric index. If the mean AUROC value is less than 0.5, this indicates very poor discriminatory power and so AUROC values are useful to compare different ROC curves and diagnostic tools.

The AUROC value that represents the best compromise between sensitivity and specificity, for any tool often the so-called turning point in the graph, is converted back to suggest a 'real' boundary value for the appropriate diagnostic tool.

Figure 4 shows the boundary values, as derived and suggested by authors themselves, for WHtR (Fig. 4(a)) and WC (Fig. 4(b)) for screening different metabolic outcomes. These thirty-five analyses arise from sixteen papers from several investigators studying populations in many different countries covering a wide age range and several ethnic $\operatorname{groups}^{(24,26,32,36,56,63,66,73,97-99,102,103,105-107)}$. Within these study populations, there were subjects with Caucasian, Asian, Afro-Caribbean and Central American ethnic backgrounds.

The mean of proposed boundary values for WHtR, weighted for study size, in men and women, respectively, was 0.52 and 0.53 for diabetes, 0.53 and 0.50 for CVD, 0.50 and 0.50 for hypertension outcomes, 0.49 and 0.49 for lipid outcomes and 0.50 and 0.49 for metabolic syndrome outcomes. The mean of all suggested boundary values for WHtR over thirty-four individual analyses covering all outcomes was 0.50 in men and 0.50 in women.

The mean of proposed boundary values for WC, weighed for study size, in men and women respectively, was 88 and $83 \mathrm{~cm}$ for diabetes, 92 and $79 \mathrm{~cm}$ for CVD, 85 and $79 \mathrm{~cm}$ for hypertension outcomes, 84 and $77 \mathrm{~cm}$ for lipid outcomes and 83 and $77 \mathrm{~cm}$ for metabolic syndrome outcomes. The mean of all suggested boundary values for WC over the same thirty-four different analyses covering all outcomes (data not shown) was for $86 \mathrm{~cm}$ in men and $79 \mathrm{~cm}$ in women.

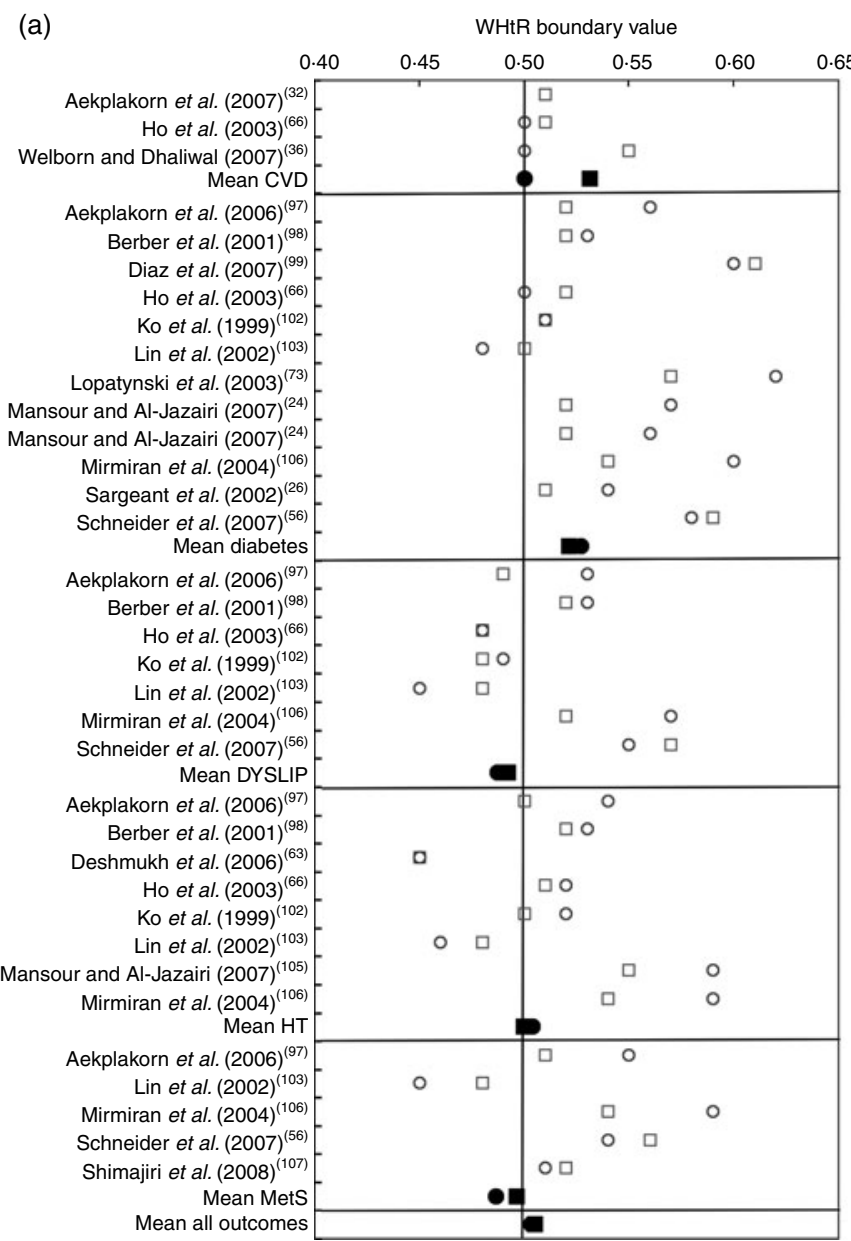

(b)

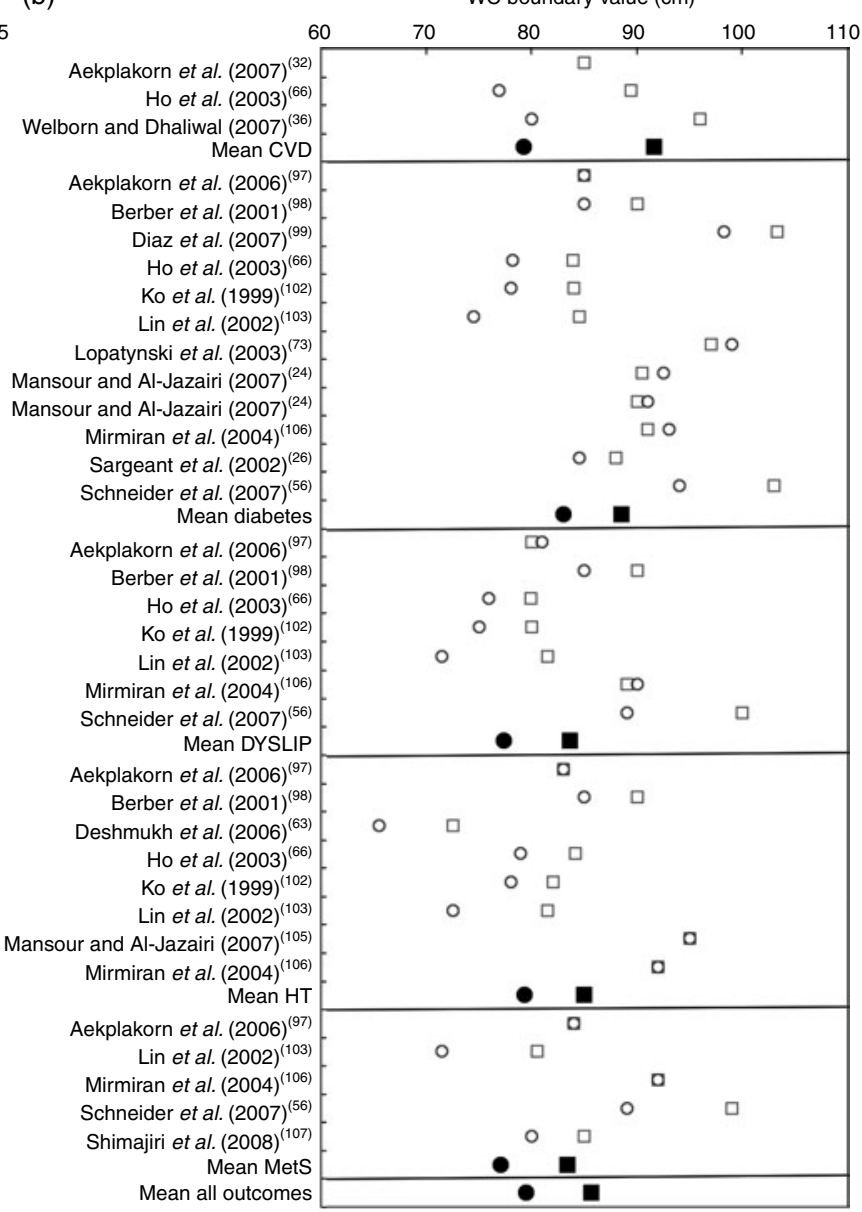

Fig. 4. Proposed boundary value for waist-to-height ratio (WHtR) (a) and waist circumference (WC) (b) from area under receiver operator characteristic (AUROC) analysis for men and women, by outcome. Mean boundary value in men ( $\square$ ) and women (O) in individual studies and as a mean by outcome in men $(\square)$ and women $(\bullet)$. Overall mean boundary values are weighted for individual study sample sizes. WC was measured at four different anatomical sites across studies; the minimum WC ${ }^{(26,98,106)}$, WC at or $1 \mathrm{~cm}$ from the umbilicus ${ }^{(24,32,73,97,105,107)}$, WC at the midpoint between the xiphisternum and the umbilicus ${ }^{(66)}$ or using the WHO definition of halfway between the lower rib and the iliac crest $^{(56,63,99,102,103)}$ The studies were performed in the following population groups: Asian ${ }^{(32,63,66,97,102,103,107)}$; Middle Eastern ${ }^{(105,106)}$; North American $^{(99)}$; Central American ${ }^{(98)}$; Caribbean ${ }^{(26)}$; European ${ }^{(56,73,99)}$. DYSLIP, dyslipidaemia; HT, hypertension; MetS, metabolic syndrome. 


\section{Discussion}

The present review is the first systematic review of the evidence supporting the use of WHtR, a proxy for abdominal fatness, as a predictor for cardiometabolic risk (i.e. predicting risk factors for CVD and diabetes). It draws on evidence from prospective and cross-sectional studies in both adults and children. Moreover, it puts the relationship between WHtR and disease into context with other proxies for obesity and abdominal obesity, namely BMI and WC. As an additional analysis, results of ROC analyses have also been summarised to indicate sensitivity and specificity of the potential predictors and to investigate possible boundary values for $\mathrm{WHtR}$.

The systematic review included data from some very large, nationally representative cohorts, in a variety of ethnic groups. Importantly, results are consistent between adults and children.

The 'summary scores' in Tables 2, 5, 6 and 8 represent our attempt, in the absence of a full meta-analysis, to summarise the outcomes of the different types of studies. We acknowledge the limitations of these 'summary scores' and that statistical significance depends on many factors such as size of the study population and the incorporation of demographic or physiological adjustment variables. Each of the three anthropometric indices is more likely to appear 'significant' in the largest study populations even though the effect size (strength) of the associations could be quite different. In cross-sectional studies, there was a tendency for very large populations to produce significant findings.

We also acknowledge that the conclusions from a systematic review can only be as good as the studies included within it. We have relied on studies published in the English language and acknowledge that publication bias may have influenced the present results. Some studies showing different results may not have been submitted for publication by their authors and some submitted studies showing different results may have been denied publication. However, the strength of any systematic review is that it is a comprehensive, transparent and an inclusive process which overcomes many other sources of bias which are sometimes found in narrative reviews.

Prospective studies in adults indicate that WHtR and WC are similarly useful as predictors of diabetes and CVD, being significant predictors with similar OR or HR. In some studies WHtR and WC have higher OR or HR than BMI or remain significant predictors after adjustment for BMI, indicating that they are possibly better predictors than BMI. Cross-sectional studies in adults and children supported the observations in prospective studies, with WHtR, WC and BMI all showing a similar proportion of significant relationships with risk factors for diabetes and CVD.

Determination of specificity and sensitivity from ROC analysis clearly showed that WHtR has high AUROC values for all the outcome measures related to diabetes and CVD. From this, we suggest that WHtR would be a good screening tool, probably better than WC (see Fig. 2).

In their paper 'Six reasons why the waist-to-height ratio is a rapid and effective global indicator for health risks of obesity and how its use could simplify the international public health message on obesity'(14) Ashwell \& Hsieh presented a narrative review of evidence to support their six reasons which they listed as follows:

(1) WHtR is more sensitive than BMI as an early warning of health risks.

(2) A boundary value of WHtR of 0.5 indicates increased risk for men and women.

(3) A boundary value of WHtR of 0.5 may indicate increased risk for individuals in different ethnic groups.

(4) WHtR is cheaper and easier to measure and calculate than BMI.

(5) WHtR may allow the same boundary values for children and adults.

(6) WHtR boundary values can be converted into a consumer-friendly chart.

Point number (1) focused on the likelihood that WHtR, as an anthropometric index which is a proxy for central obesity, could be more useful than BMI in the prediction of health risks (the word sensitive was not used in relation to any particular boundary value in this case). The present systematic review has provided supportive evidence for this point, with WHtR (and WC) being a risk factor more often than BMI. Further, the AUROC analysis has provided good evidence that WHtR is probably a better diagnostic predictor than BMI or WC. To provide further statistical support for this statement a meta-analysis is required. However, this is beyond the scope of the present systematic review. The AUROC data have also provided good evidence that the suggestion made in point number (2) that a WHtR of 0.5 is a good boundary value for men and women across many ethnic groups. Comparison of the summary lines covering all health outcomes in Fig. 4(a) and (b) and the suggested mean boundary values for WHtR and WC show clearly that the same boundary value of WHtR can be used for men and women (0.5), whilst distinct WC boundary values must be used.

Thus, these analyses support the proposal that WHtR may be advantageous because it avoids the need for age-, sex- and ethnic-specific boundary values ${ }^{(14)}$ and helps to avoid the confusion whereby many different boundary values for WC have been published for different ethic groups ${ }^{(107)}$. If the suggested boundary value of WHtR 0.5 were to be adopted, this would simplify the application of this diagnostic tool to provide the health message 'keep your waist circumference to less than half your height'. Further studies are required in men, women and children of different ethnic groups to add support to point number (5).

In relation to point (4), WC can be measured more cheaply and less intrusively than BMI, since measuring weight requires accurate scales and often requires some degree of subject undressing. However, it is recognised that WC can be measured at a variety of different sites (indicated in the legend for Fig. 4(a) and (b)) and that this will affect the boundary value. Importantly, comparison of WC measured at a variety of sites indicates that all are highly reproducible and are similarly correlated with total body fat in a sex-dependent manner ${ }^{(108)}$. While measuring the narrowest WC would be expected to give smaller values of WC than other techniques, it is interesting in Fig. 4(a) and (b) that the studies using this method did not give the smallest boundary value for WHtR or for WC. 
In relation to point number (6), the principle of a Shape Chart, to replace a Weight Chart, was proposed as early as $1995^{(109)}$. Prototypes of charts for adults based on boundary values for WHtR were suggested shortly afterwards ${ }^{(110,111)}$, followed by the publication in $2005^{(14)}$. The AUROC data have confirmed that 0.5 is an appropriate boundary value for increased risk in men and women from many ethnic groups. Further corroboration of 0.6 , originally proposed pragmatically as a boundary value for higher risk ${ }^{(111)}$, and the appropriate boundary value for risk in children, awaits further studies.

\section{Final conclusions}

Observations of seventy-eight prospective and crosssectional studies suggest that while WHtR, WC, and BMI are all predictors of CVD, diabetes and related risk factors, WHtR and WC are more probably reliable predictors than BMI. A meta-analysis is now required to provide further statistical support for these suggestions. The AUROC analysis and the calculation of a weighted mean WHtR of 0.5 suggests that WHtR is a suitable screening tool applicable to a wide variety of populations. Its simplicity and its conversion to the easily remembered public health message 'keep your waist circumference to less than half your height' argues for its practical adoption.

It is pleasing that various authoritative bodies recognise the importance of central obesity by recommending the use of $\mathrm{WC}$ as a useful screening tool in many primary care situations. However, there is the problem that at least five different cut-off levels of WC, for different sexes, ethnic groups and even different countries, have been proposed (summarised in Alberti et al. $2009^{(112)}$ ) to account for the effect of height on metabolic risk within different populations. The use of WHtR, with a simple global boundary value of $0 \cdot 5$, could overcome this confusing situation, with obvious benefit to the public health promotion message.

The early phase of the systematic literature search indicated that the number of papers that reported relationships between WHtR and health outcomes is miniscule in the context of those which report other simple obesity measures and health outcomes: they are only $2 \%$ of WC papers, and $0.2 \%$ of BMI papers. It is, perhaps, surprising that, up to the cut-off point for our systematic review (end of 2008), as many as seventy-eight 'included' papers had calculated WHtR as well as WC when they were investigating the effect of central obesity on a variety of health outcomes. We have noticed a substantial increase in WHtR papers in 2009 and the first part of $2010^{(113-118)}$, many of which provide data to support the use of the boundary value of 0.5 , or use 0.5 as the boundary value to divide their population and assess risk. We hope that the evidence summarised here for $\mathrm{WHtR}$ as a predictor of diabetes, CVD and related risk factors, and the usefulness of the global boundary value of $0 \cdot 5$, will encourage the use of this index in existing and future studies. This would provide more data and allow further consideration and confirmation of clinically relevant boundary values for WHtR in children as well as adults.

\section{Acknowledgements}

This research received no specific grant from any funding agency in the public, commercial or not-for-profit sectors.

L. M. B. was involved in study design, collected the data and prepared the manuscript, M. A. and S. D. H. were involved in study design, preparation and review of the manuscript.

There are no conflicts of interest to declare.

\section{References}

1. Soto Gonzalez A, Bellido D, Buno MM, et al. (2007) Predictors of the metabolic syndrome and correlation with computed axial tomography. Nutrition 23, 36-45.

2. Vague J (1946) Le traitment des obesities (Treatment of obesity). Marseille Med 83, 210-225.

3. Vague $\mathbf{J}$ (1956) The degree of masculine differentiation of obesities: a factor determining predisposition to diabetes, atherosclerosis, gout, and uric calculous disease. Am J Clin Nutr 4, 20-34.

4. Ashwell M, Cole TJ \& Dixon AK (1985) Obesity: new insight into the anthropometric classification of fat distribution shown by computed tomography. BMJ 290, 1692-1694.

5. Seidell JC, Cigolini M, Charzewska J, et al. (1990) Fat distribution in European women: a comparison of anthropometric measurements in relation to cardiovascular risk factors. Int J Epidemiol 19, 303-308.

6. Bjorntorp P (1988) The associations between obesity, adipose tissue distribution and disease. Acta Med Scand 723, $121-134$.

7. Han TS, van Leer EM, Seidell JC, et al. (1995) Waist circumference action levels in the identification of cardiovascular risk factors: prevalence study in a random sample. BMJ 311, $1401-1405$.

8. Hsieh SD \& Yoshinaga H (1995) Is there any difference in coronary heart disease risk factors and prevalence of fatty liver in subjects with normal body mass index having different physiques? Tohoku J Exp Med 177, 223-231.

9. Hsieh SD \& Yoshinaga H (1995) Waist/height ratio as a simple and useful predictor of coronary heart disease risk factors in women. Inter Med 34, 1147-1152.

10. Hsieh SD \& Yoshinaga H (1995) Abdominal fat distribution and coronary heart disease risk factors in men-waist/height ratio as a simple and useful predictor. Int J Obes 19, 585-589.

11. Ashwell M, Lejeune S \& McPherson K (1996) Ratio of waist circumference to height may be better indicator of need for weight management. BMJ 312, 377.

12. Lee JS, Aoki K, Kawakubo K, et al. (1995) A study on indices of body fat distribution for screening for obesity. Sangyo Eiseigaku Zasshi 37, 9-18.

13. Ashwell M, Cole TJ \& Dixon AK (1996) Ratio of waist circumference to height is strong predictor of intraabdominal fat. BMJ 313, 559-560.

14. Ashwell M \& Hsieh SD (2005) Six reasons why the waist-to-height ratio is a rapid and effective global indicator for health risks of obesity and how its use could simplify the international public health message on obesity. Int $J$ Food Sci Nutr 56, 303-307.

15. World Health Organization (1998) Obesity: Preventing and Managing the Global Epidemic. Report of a WHO Consultation on Obesity. Geneva: WHO, Geneva 3-5 June 1997. 
16. Fasanmade OA \& Okubadejo NU (2007) Magnitude and gender distribution of obesity and abdominal adiposity in Nigerians with type 2 diabetes mellitus. Niger J Clin Pract 10, $52-57$.

17. Moreira-Andres MN, del Canizo-Gomez FJ, Losa MA, et al. (2004) Comparison of anthropometric parameters as predictors of serum lipids in premenopausal women. J Endocrinol Invest 27, 340-347.

18. Yasein NA, Mas'ad DF, Al-Zaru L, et al. (2008) The prediction of diabetes and hypertension by anthropometric indices based on family medicine clinic data at Jordan University Hospital. J Bahrain Med Soc 20, 8-14.

19. Al-Mahroos F \& McKeigue P (1998) Obesity, physical activity and prevalence of diabetes in Bahraini Arab native population. Bahrain Med Bull 20, 114-118.

20. Bray GA, Jablonski KA, Fujimoto WY, et al. (2008) Relation of central adiposity and body mass index to the development of diabetes in the Diabetes Prevention Program. Am J Clin Nutr 87, 1212-1218.

21. Chei C-L, Iso H, Yamagishi K, et al. (2008) Body fat distribution and the risk of hypertension and diabetes among Japanese men and women. Hypertens Res 31, 851-857.

22. Hadaegh F, Zabetian A, Harati H, et al. (2006) Waist/height ratio as a better predictor of type 2 diabetes compared to body mass index in Tehranian adult men - a 3.6-year prospective study. Exp Clin Endocrinol Diabetes 114, $310-315$.

23. MacKay M, Haffner S, Wagenknecht L, et al. (2009) Prediction of type 2 diabetes using alternative anthropometric measures in a multi-ethnic cohort: The Insulin Resistance Atherosclerosis Study. Diabetes Care 32, 956-958.

24. Mansour AA \& Al-Jazairi MI (2007) Predictors of incident diabetes mellitus in Basrah, Iraq. Ann Nutr Metab 51, 277-280.

25. Nyamdorj R, Qiao Q, Soderberg S, et al. (2009) BMI compared with central obesity indicators as a predictor of diabetes incidence in Mauritius. Obesity (Silver Spring) 17, 342-348.

26. Sargeant LA, Bennett FI, Forrester TE, et al. (2002) Predicting incident diabetes in Jamaica: the role of anthropometry. Obes Res 10, 792-798.

27. Anonymous (2006) Relationship of body size and shape to the development of diabetes in the diabetes prevention program. Obesity (Silver Spring) 14, 2107-2117.

28. Tulloch-Reid MK, Williams DE, Looker HC, et al. (2003) Do measures of body fat distribution provide information on the risk of type 2 diabetes in addition to measures of general obesity? Comparison of anthropometric predictors of type 2 diabetes in Pima Indians. Diabetes Care 26, 2556-2561.

29. Gelber RP, Gaziano JM, Orav EJ, et al. (2008) Measures of obesity and cardiovascular risk among men and women. $J$ Am Coll Cardiol 52, 605-615.

30. Zhang X, Shu XO, Gao YT, et al. (2009) General and abdominal adiposity and risk of stroke in Chinese women. Stroke 40, 1098-1104.

31. Zhang X, Shu XO, Gao YT, et al. (2004) Anthropometric predictors of coronary heart disease in Chinese women. Int J Obes 28, 734-740.

32. Aekplakorn W, Pakpeankitwatana V, Lee CMY, et al. (2007) Abdominal obesity and coronary heart disease in Thai men. Obesity 15, 1036-1042.

33. Cox BD, Whichelow MJ \& Prevost AT (1998) The development of cardiovascular disease in relation to anthropometric indices and hypertension in British adults. Int J Obes 22, 966-973.
34. Page JH, Rexrode KM, Hu F, et al. (2009) Waist-height ratio as a predictor of coronary heart disease among women. Epidemiology 20, 361-366.

35. Pischon T, Boeing H, Hoffmann K, et al. (2008) General and abdominal adiposity and risk of death in Europe. $\mathrm{N} \mathrm{Engl}$ $J$ Med 359, 2105-2120.

36. Welborn T \& Dhaliwal S (2007) Preferred clinical measures of central obesity for predicting mortality. Eur J Clin Nutr 61, 1373-1379.

37. Wessel TR, Arant CB, Olson MB, et al. (2004) Relationship of physical fitness vs body mass index with coronary artery disease and cardiovascular events in women. JAMA 292 , 1179-1187.

38. Lu M, Ye W, Adami HO, et al. (2006) Prospective study of body size and risk for stroke amongst women below age 60. J Intern Med 260, 442-450.

39. Fuchs FD, Gus M, Moreira LB, et al. (2005) Anthropometric indices and the incidence of hypertension: a comparative analysis. Obes Res 13, 1515-1517.

40. Nyamdorj R, Qiao Q, Söderberg S, et al. (2008) Comparison of body mass index with waist circumference, waist-to-hip ratio, and waist-to-stature ratio as a predictor of hypertension incidence in Mauritius. J Hypertens 26, $866-870$.

41. Panagiotakos DB, Chrysohoou C, Pitsavos C, et al. (2009) Hierarchical analysis of anthropometric indices in the prediction of 5-year incidence of hypertension in apparently healthy adults: The ATTICA study. Atherosclerosis 206, 314-320.

42. Gaglione MM, Can AS, Schneider HJ, et al. (2009) Obesity and risk of death. N Engl J Med 360, 1042-1044.

43. Ajay VS, Prabhakaran D, Jeemon P, et al. (2008) Prevalence and determinants of diabetes mellitus in the Indian industrial population. Diabet Med 25, 1187-1194.

44. Azizi F, Esmaillzadeh A \& Mirmiran P (2004) Obesity and cardiovascular disease risk factors in Tehran adults: a population-based study. East Mediterr Health J 10, 887-897.

45. Bertsias G, Mammas I, Linardakis M, et al. (2003) Overweight and obesity in relation to cardiovascular disease risk factors among medical students in Crete, Greece. BMC Public Health 3, 3.

46. Brouwer BG, Visseren FL, Stolk RP, et al. (2007) Abdominal fat and risk of coronary heart disease in patients with peripheral arterial disease. Obesity (Silver Spring) 15, $1623-1630$.

47. Cox BD, Whichelow MJ, Ashwell M, et al. (1997) Association of anthropometric indices with elevated blood pressure in British adults. Int J Obes 21, 674-680.

48. Esmaillzadeh A, Mirmiran P \& Azizi F (2004) Waist-to-hip ratio is a better screening measure for cardiovascular risk factors than other anthropometric indicators in Tehranian adult men. Int J Obes 28, 1325-1332.

49. Esmaillzadeh A, Mirmiran P \& Azizi F (2006) Comparative evaluation of anthropometric measures to predict cardiovascular risk factors in Tehranian adult women. Public Health Nutr 9, 61-69.

50. Harris MM, Stevens J, Thomas N, et al. (2000) Associations of fat distribution and obesity with hypertension in a bi-ethnic population: the ARIC study, Atherosclerosis Risk in Communities Study. Obesity Res 8, 516-524.

51. He Y, Zhai F, Ma G, et al. (2008) Abdominal obesity and the prevalence of diabetes and intermediate hyperglycaemia in Chinese adults. Public Health Nutr 12, 1078-1084.

52. Hsieh SD, Yoshinaga H, Muto T, et al. (2000) Health risks among Japanese men with moderate body mass index. Int $J$ Obesity 24, 358-362. 
53. Jeong S-K, Seo M-W, Kim Y-H, et al. (2005) Does waist indicate dyslipidemia better than BMI in Korean adult population? J Korean Med Sci 20, 7-12.

54. Sakurai M, Miura K, Takamura T, et al. (2006) Gender differences in the association between anthropometric indices of obesity and blood pressure in Japanese. Hypertens Res 29, 75-80.

55. Sayeed MA, Mahtab H, Latif ZA, et al. (2003) Waist-toheight ratio is a better obesity index than body mass index and waist-to-hip ratio for predicting diabetes, hypertension and lipidemia. Bangladesh Med Res Counc Bull 29, 1-10.

56. Schneider HJ, Glaesmer H, Klotsche J, et al. (2007) Accuracy of anthropometric indicators of obesity to predict cardiovascular risk. J Clin Endocrin Metab 92, 589-594.

57. Tseng CH (2008) Waist-to-height ratio and coronary artery disease in Taiwanese type 2 diabetic patients. Obesity (Silver Spring) 16, 2754-2759.

58. Wang Z, Rowley K, Wang Z, et al. (2007) Anthropometric indices and their relationship with diabetes, hypertension and dyslipidemia in Australian Aboriginal people and Torres Strait Islanders. Eur J Cardiovasc Prev Rehabil 14, $172-178$.

59. Wu H-Y, Chen L-L, Zheng J, et al. (2007) Simple anthropometric indices in relation to cardiovascular risk factors in Chinese type 2 diabetic patients. Chin J Physiol 50, $135-142$.

60. Bosy-Westphal A, Geisler C, Onur S, et al. (2006) Value of body fat mass vs anthropometric obesity indices in the assessment of metabolic risk factors. Int $J$ Obes 30, 475-483.

61. Can AS, Bersot TP \& Gonen M (2009) Anthropometric indices and their relationship with cardiometabolic risk factors in a sample of Turkish adults. Public Health Nutr 12, 538-546.

62. Chehrei A, Sadrnia S, Keshteli AH, et al. (2007) Correlation of dyslipidemia with waist to height ratio, waist circumference, and body mass index in Iranian adults. Asia Pac J Clin Nutr 16, 248-253.

63. Deshmukh PR, Gupta SS, Dongre AR, et al. (2006) Relationship of anthropometric indicators with blood pressure levels in rural Wardha. Indian J Med Res 123, 657-664.

64. Ghosh J \& Bandyopadhyay A (2007) Comparative evaluation of obesity measures: relationship with blood pressures and hypertension. Singapore Med J 48, 232-235.

65. Gracey M, Burke V, Martin DD, et al. (2007) Assessment of risks of 'lifestyle' diseases including cardiovascular disease and type 2 diabetes by anthropometry in remote Australian Aborigines. Asia Pac J Clin Nutr 16, 688-697.

66. Ho SY, Lam TH \& Janus ED (2003) Waist to stature ratio is more strongly associated with cardiovascular risk factors than other simple anthropometric indices. Ann Epidemiol 13, 683-691.

67. Hsieh SD, Yoshinaga H \& Muto T (2003) Waist-to-height ratio, a simple and practical index for assessing central fat distribution and metabolic risk in Japanese men and women. Int J Obes 27, 610-616.

68. Hsieh SD \& Muto T (2005) The superiority of waist-toheight ratio as an anthropometric index to evaluate clustering of coronary risk factors among non-obese men and women. Prev Med 40, 216-220.

69. Kaur P, Radhakrishnan E, Sankarasubbiyan S, et al. (2008) A comparison of anthropometric indices for predicting hypertension and type 2 diabetes in a male industrial population of Chennai, South India. Ethn Dis 18, 31-36.
70. Khan A, Haq FU, Pervez MB, et al. (2008) Anthropometric correlates of blood pressure in normotensive Pakistani subjects. Int J Cardiol 124, 259-262.

71. Kotchen TA, Grim CE, Kotchen JM, et al. (2008) Altered relationship of blood pressure to adiposity in hypertension. Am J Hypertens 21, 284-289.

72. Lee K, Song Y-M \& Sung J (2008) Which obesity indicators are better predictors of metabolic risk? Healthy Twin Study. Obesity 16, 834-840.

73. Lopatynski J, Mardarowicz G \& Szczesniak G (2003) A comparative evaluation of waist circumference, waist-tohip ratio, waist-to-height ratio and body mass index as indicators of impaired glucose tolerance and as risk factors for type-2 diabetes mellitus. Ann Univ Mariae Curie Sklodowska Med 58, 413-419.

74. Lovegrove JA, Silva KDRR, Wright JW, et al. (2002) Adiposity, insulin and lipid metabolism in post-menopausal women. Int J Obes 26, 475-486.

75. Maher V, O'Dowd M, Carey M, et al. (2009) Association of central obesity with early carotid intima-media thickening is independent of that from other risk factors. Int $J$ Obes 33, 136-143.

76. Mukuddem-Petersen J, Snijder MB, van Dam RM, et al. (2006) Sagittal abdominal diameter: no advantage compared with other anthropometric measures as a correlate of components of the metabolic syndrome in elderly from the Hoorn Study. Am J Clin Nutr 84, 995-1002.

77. Paniagua L, Lohsoonthorn V, Lertmaharit S, et al. (2008) Comparison of waist circumference, body mass index, percent body fat and other measure of adiposity in identifying cardiovascular disease risks among Thai adults. Obes Res Clin Pract 2, 215-223.

78. Patel S, Unwin N, Bhopal R, et al. (1999) A comparison of proxy measures of abdominal obesity in Chinese, European and South Asian adults. Diabet Med 16, 853-860.

79. Rissanen P, Hamalainen P, Vanninen E, et al. (1997) Relationship of metabolic variables to abdominal adiposity measured by different anthropometric measurements and dual-energy X-ray absorptiometry in obese middle-aged women. Int J Obes 21, 367-371.

80. Sattar N, Tan CE, Han TS, et al. (1998) Associations of indices of adiposity with atherogenic lipoprotein subfractions. Int J Obes 22, 432-439.

81. Thomas GN, Young RP, Tomlinson B, et al. (1999) A sibling-pair analysis of fasting lipids and anthropometric measurements and their relationship to hypertension. Clin Exp Hypertens 21, 1161-1176.

82. Turcato E, Bosello O, Di Francesco V, et al. (2000) Waist circumference and abdominal sagittal diameter as surrogates of body fat distribution in the elderly: their relation with cardiovascular risk factors. Int J Obes 24, 1005-1010.

83. Yasmin \& Mascie-Taylor CG (2000) Adiposity indices and their relationship with some risk factors of coronary heart disease in middle-aged Cambridge men and women. Ann Hum Biol 27, 239-248.

84. Botton J, Heude B, Kettaneh A, et al. (2007) Cardiovascular risk factor levels and their relationships with overweight and fat distribution in children: the Fleurbaix Laventie Ville Sante II study. Metabolism 56, 614-622.

85. Hara M, Saitou E, Iwata F, et al. (2002) Waist-to-height ratio is the best predictor of cardiovascular disease risk factors in Japanese schoolchildren. J Atheroscler Thromb $\mathbf{9}$, $127-132$

86. Manios Y, Kourlaba G, Kafatos A, et al. (2008) Associations of several anthropometric indices with insulin resistance in children: The Children Study. Acta Paediatr 97, 494-499. 
87. Mirzaei M, Taylor R, Morrell S, et al. (2007) Predictors of blood pressure in a cohort of school-aged children. Eur J Cardiovasc Prev Rehabil 14, 624-629.

88. Ruiz JR, Ortega FB, Loit HM, et al. (2007) Body fat is associated with blood pressure in school-aged girls with low cardiorespiratory fitness: the European Youth Heart Study. J Hypertens 25, 2027-2034.

89. Sung RYT, Yu CCW, Choi KC, et al. (2007) Waist circumference and body mass index in Chinese children: cutoff values for predicting cardiovascular risk factors. Int $J$ Obes 31, 550-558.

90. Teixeira PJ, Sardinha LB, Going SB, et al. (2001) Total and regional fat and serum cardiovascular disease risk factors in lean and obese children and adolescents. Obes Res 9, $432-442$.

91. Freedman DS, Dietz WH, Srinivasan SR, et al. (2009) Risk factors and adult body mass index among overweight children: The Bogalusa Heart Study. Pediatrics 123, 750-757.

92. Garnett SP, Baur LA \& Cowell CT (2008) Waist-to-height ratio: a simple option for determining excess central adiposity in young people. Int J Obes 32, 1028-1030.

93. Maffeis C, Banzato C, Talamini G, et al. (2008) Waist-toheight ratio, a useful index to identify high metabolic risk in overweight children. J Pediatr 152, 207-213.

94. Savva SC, Tornaritis M, Savva ME, et al. (2000) Waist circumference and waist-to-height ratio are better predictors of cardiovascular disease risk factors in children than body mass index. Int J Obes 24, 1453-1458.

95. Kahn HS, Imperatore G \& Cheng YJ (2005) A populationbased comparison of BMI percentiles and waist-to-height ratio for identifying cardiovascular risk in youth. J Pediatr 146, $482-488$.

96. Mesa JL, Ortega FB, Ruiz JR, et al. (2006) Anthropometric determinants of a clustering of lipid-related metabolic risk factors in overweight and non-overweight adolescents - influence of cardiorespiratory fitness. The Avena Study. Ann Nutr Metab 50, 519-527.

97. Aekplakorn W, Kosulwat V \& Suriyawongpaisal P (2006) Obesity indices and cardiovascular risk factors in Thai adults. Int J Obes 30, 1782-1790.

98. Berber A, Gomez-Santos R, Fanghanel G, et al. (2001) Anthropometric indexes in the prediction of type 2 diabetes mellitus, hypertension and dyslipidaemia in a Mexican population. Int J Obes 25, 1794-1799.

99. Diaz VA, Mainous AG III, Baker R, et al. (2007) How does ethnicity affect the association between obesity and diabetes? Diabet Med 24, 1199-1204.

100. Freedman DS, Kahn HS, Mei Z, et al. (2007) Relation of body mass index and waist-to-height ratio to cardiovascular disease risk factors in children and adolescents: the Bogalusa Heart Study. Am J Clin Nutr 86, 33-40.

101. Hsieh SD \& Muto T (2006) Metabolic syndrome in Japanese men and women with special reference to the anthropometric criteria for the assessment of obesity: proposal to use the waist-to-height ratio. Prev Med 42, $135-139$.

102. Ko GT, Chan JC, Cockram CS, et al. (1999) Prediction of hypertension, diabetes, dyslipidaemia or albuminuria using simple anthropometric indexes in Hong Kong Chinese. Int J Obes 23, 1136-1142.

103. Lin WY, Lee LT, Chen CY, et al. (2002) Optimal cut-off values for obesity: using simple anthropometric indices to predict cardiovascular risk factors in Taiwan. Int $J$ Obesity 26, $1232-1238$.

104. Lorenzo C, Serrano-Rios M, Martinez-Larrad MT, et al. (2007) Which obesity index best explains prevalence differences in type 2 diabetes mellitus? Obesity 15, 1294-1301.

105. Mansour AA \& Al-Jazairi MI (2007) Cut-off values for anthropometric variables that confer increased risk of type 2 diabetes mellitus and hypertension in Iraq. Arch Med Res 38, 253-258.

106. Mirmiran P, Esmaillzadeh A \& Azizi F (2004) Detection of cardiovascular risk factors by anthropometric measures in Tehranian adults: receiver operating characteristic (ROC) curve analysis. Eur J Clin Nutr 58, 1110-1118.

107. Shimajiri T, Imagawa M, Kokawa M, et al. (2008) Revised optimal cut-off point of waist circumference for the diagnosis of metabolic syndrome in Japanese women and the influence of height. J Atheroscler Thromb 15, 94-99.

108. Wang J, Thornton J, Bari S, et al. (2003) Comparisons of waist circumferences measured at 4 sites. Am J Clin Nutr 77, 379-384.

109. Ashwell MA (1995) A new shape chart for assessing the risks of obesity. Proc Nutr Soc 54, 86A.

110. Ashwell M (1997) The Ashwell Shape Chart - a new millennium approach to communicate the metabolic risks of obesity. Obes Res 5, 45S.

111. Ashwell M (1998) The Ashwell Shape Chart - a public health approach to the metabolic risks of obesity. Int J Obes 22, Suppl. 3, S213.

112. Alberti KGMM, Eckel RH, Grundy SM, et al. (2009) Harmonizing the metabolic syndrome: a joint interim statement of the International Diabetes Federation Task Force on Epidemiology and Prevention; National Heart, Lung, and Blood Institute; American Heart Association; World Heart Federation; International Atherosclerosis Society; and International Association for the Study of Obesity. Circulation 120, 1640-1645.

113. Ashwell MA \& Gibson S (2009) Waist to height ratio is a simple and effective obesity screening tool for cardiovascular risk factors: analysis of data from the British National Diet and Nutrition Survey of adults aged 19-64 years. Obes Facts 2, 97-103.

114. Schneider HJ, Friedrich N, Klotsche J, et al. (2010) The predictive value of different measures of obesity for incident cardiovascular events and mortality. J Clin Endocrinol Metab 95, 1777-1785.

115. Taylor AW, Ibrahim S, Ben-Shlomo Y, et al. (2010) Comparison of the associations of body mass index and measures of central adiposity and fat mass with coronary heart disease, diabetes, and all-cause mortality: a study using data from 4 UK cohorts. Am J Clin Nutr 91, 547-556.

116. Goulding A, Taylor RW, Grant AM, et al. (2010) Waist-toheight ratios in relation to BMI z-scores in three ethnic groups from a representative sample of New Zealand children aged 5-14 years. Int J Obesity 34, 1188-1190.

117. Nambiar S, Hughes I \& Davies PS (2010) Developing waistto-height ratio cut-offs to define overweight and obesity in children and adolescents. Public Health Nutr 13, $1566-1574$

118. Xiong F, Garnett SP, Cowell CT, et al. (2010) Waist circumference and waist-to-height ratio in Han Chinese children living in Chongqing south-west China. Public Health Nutr (epublication ahead of print version 18 March 2010). 